\title{
Report
}

\section{NIST-TAPPI Workshop on Measurement Needs for Cellulose Nanomaterials}

\author{
Fairmont Hotel, Vancouver, Canada
}

23 June 2014

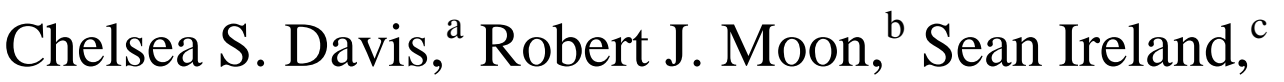

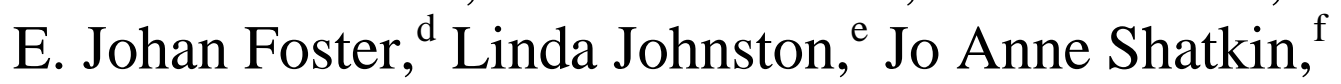
Kim Nelson, ${ }^{\mathrm{g}}$ Aaron M. Forster, ${ }^{\mathrm{a}}$ Michael T. Postek, András E. Vladár, Jeffrey W. Gilman ${ }^{\mathrm{a}}$
\end{abstract}

${ }^{a}$ National Institute of Standards and Technology, ${ }^{b}$ Forest Service, Forest Products Laboratory, ${ }^{\mathrm{c}}$ Verso Corporation, ${ }^{\mathrm{d}}$ Virginia Polytechnic Institute and State University, ${ }^{\mathrm{e}}$ National Research Council Canada, ${ }^{\mathrm{f}}$ Vireo Advisors, LLC, ${ }^{\mathrm{g}}$ American Process, Inc.

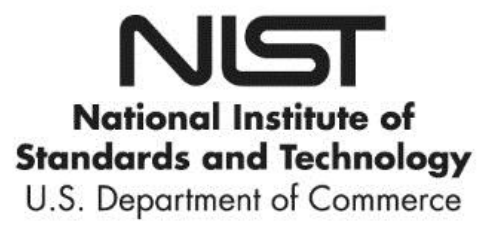

National Institute of Standards and Technology

Gaithersburg, MD 20899

USA

June 2015

DOI: 10.6028/NIST.SP.1192 
Report NIST-TAPPI Workshop on Measurement Needs for Cellulose Nanomaterials Fairmont Hotel, Vancouver, Canada 23 June 2014

Chelsea S. Davis

Jeffrey W. Gilman

Materials Science and Engineering Division

Material Measurement Laboratory

Robert J. Moon

Forest Products Laboratory

USDA Forest Service

Sean Ireland

Verso Corporation

Aaron M. Forster

Materials and Structural Systems Division

Engineering Laboratory
Michael T. Postek

András E. Vladár

Semiconductor and Dimensional Metrology

Division

Physical Measurement Laboratory

E. Johan Foster

Virginia Polytechnic Institute and State

University

Linda Johnston

National Research Council Canada

Jo Anne Shatkin

Vireo Advisers, LLC

Kim Nelson

American Process, Inc.

This publication is available free of charge from:

http://dx.doi.org/10.6028/NIST.SP.1192

June 2015

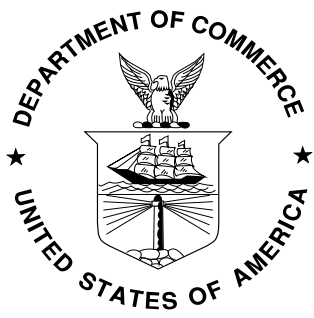

U.S. Department of Commerce Penny Pritzker, Secretary

National Institute of Standards and Technology Willie May, Under Secretary of Commerce for Standards and Technology and Director 


\section{Disclaimer:}

Certain commercial entities, equipment or materials may be identified in this document in order to describe an experimental procedure or concept adequately. Such identification is not intended to imply recommendation or endorsement by the National Institute of Standards and Technology, nor is it intended to imply that the entities, materials or equipment are necessarily the best available for the purpose.

National Institute of Standards and Technology Special Publication 1192 Natl. Inst. Stand. Technol. Spec. Publ. 1192, 42 pages (June 2015) CODEN: NSPUE2

This publication is available free of charge from: http://dx.doi.org/10.6028/NIST.SP.1192 


\section{Acknowledgements}

The organizers would like to thank the 2014 Technical Association of the Pulp and Paper Industry (TAPPI) International Conference on Nanotechnology for Renewable Materials cochairs Wadood Hamad, Akira Isogai, and Orlando Rojas for graciously allowing the Measurement Needs for Cellulose Nanomaterials (MNCNM) Workshop to be held in conjunction with the annual conference. The TAPPI staff, particularly Colleen Walker and Libby Settle, was instrumental in organizing and coordinating the logistics and registration of the MNCNM Workshop.

We also acknowledge the National Institute of Standards and Technology (NIST) Measurement Needs for Cellulose Nanomaterials Workshop speakers: Alan Rudie, Kim Nelson, Robert Moon, Sean Ireland, Linda Johnston, Wadood Hamad, Mike Bilodeau, Johan Foster, Jo Anne Shatkin, and Aaron Forster. By contributing their time and expertise, the workshop was very successful and productive.

The reporters of the Workshop Breakout Sessions, Alan Rudie, Johan Foster, and World Nieh aided in the coordination and summary of the three afternoon sessions.

Finally, we would like to thank Jeffrey Youngblood, a professor in the School of Materials Engineering at Purdue University, for his external review of the final workshop report. Muzhou "Mitchell" Wang, a National Research Council Postdoctoral Fellow at NIST also provided critical feedback on the manuscript. 


\section{Table of Contents}

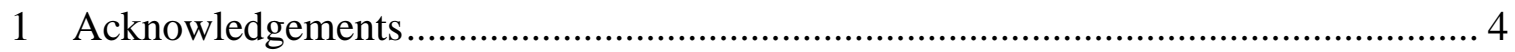

2 Table of Contents ....................................................................................... 5

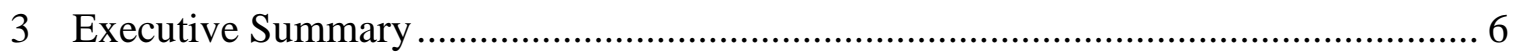

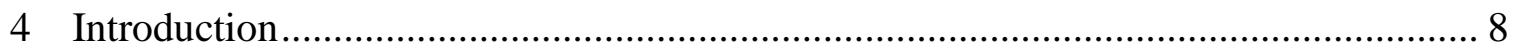

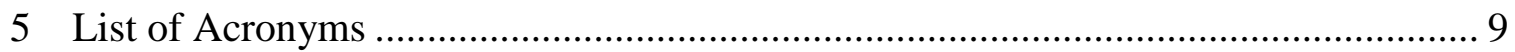

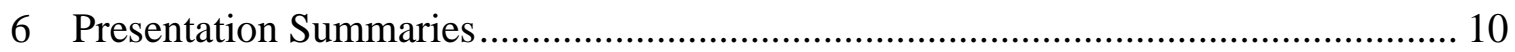

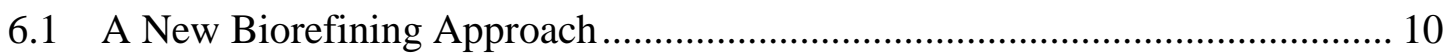

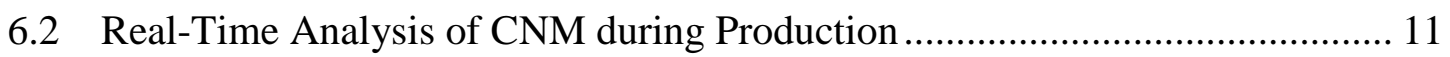

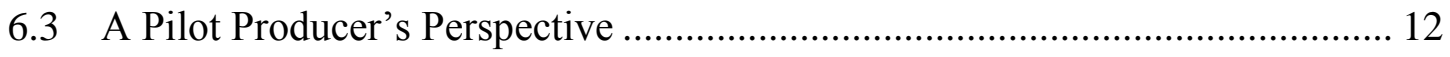

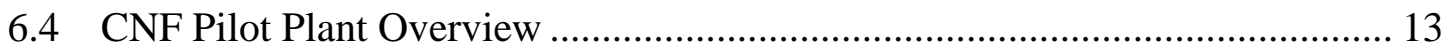

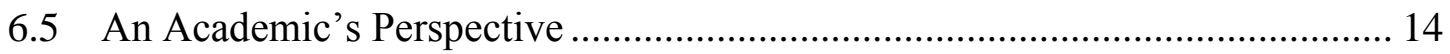

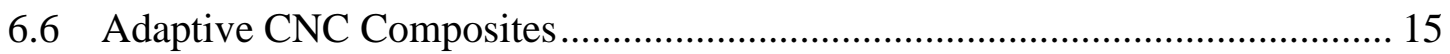

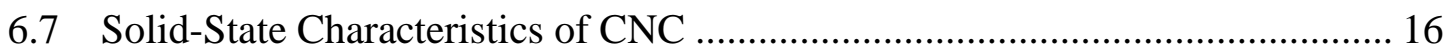

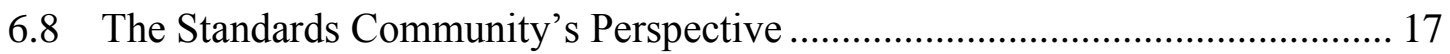

6.9 Service Life Prediction for CNM Composites.............................................. 19

6.10 CNM Environmental Health and Safety Roadmap...................................... 21

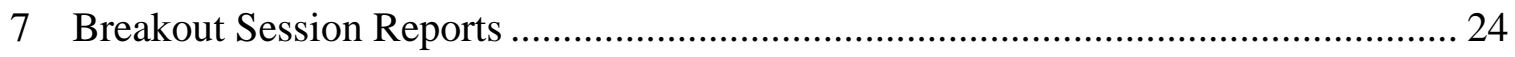

7.1 Manufacturing Breakout Session Breakout Session Summary ....................... 24

7.2 Research and Development Breakout Session Summary ............................... 26

7.3 Standards Breakout Session Summary ........................................................... 29

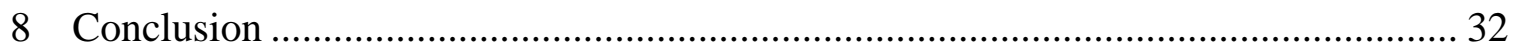

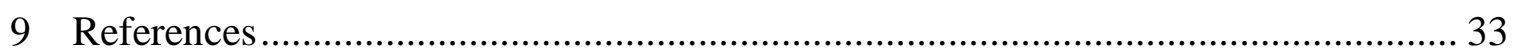

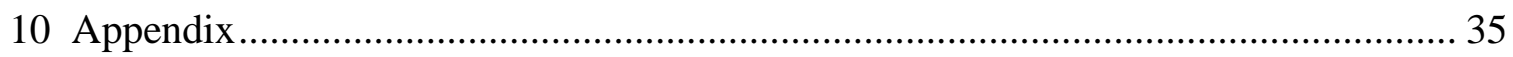

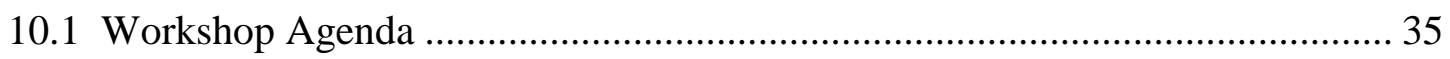

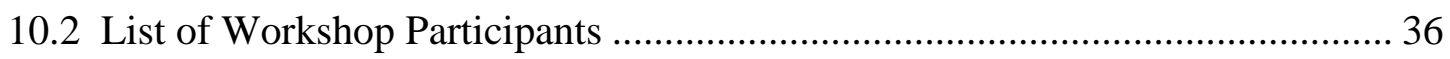

10.3 ISO TC 229 Project 19716 - Characterization of Cellulose Nanocrystals......... 37

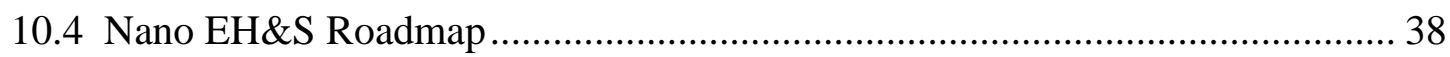




\section{Executive Summary}

A one-day workshop focused on the Measurement Needs for Cellulosic Nanomaterials was organized by the National Institute of Standards and Technology and held in conjunction with the 2014 Technical Association of the Pulp and Paper Industry (TAPPI) International Conference on Nanotechnology for Renewable Materials in Vancouver, British Columbia, Canada on June 23, 2014. The workshop consisted of presentations by leaders from industry, academia, and standards policy groups who work with nanocellulosic materials. In addition, three breakout sessions, focused on manufacturing, research and development, and standards development, were held to allow for more pointed and in depth discussion of specific measurement needs within each community. The workshop was attended by 31 registered participants. Approximately ten attendees participated in each breakout session.

Workshop speakers were organized according to expertise in the breakout session topics and tasked with providing an overview of critical needs for each topic area. Instrument manufacturers were invited to provide their perspective on the development of new metrologies, which could be used in the manufacture of cellulose nanomaterials (CNM)-based products.

The results from the workshop clearly identified barriers to innovation related to a number of significant measurement challenges. The critical measurement needs for processing nanocellulose were sorted into four characterization categories: (1) geometry, (2) surface chemistry, (3) processing, and (4) environmental health impacts. The most critical geometry measurements were fast molecular level resolution of particle size and morphology. In particular, degree of branching and/or fibrillation for nanofibrillated cellulose (CNF) and aspect ratio for cellulosic nanocrystals (CNC) must be assessed. Chemistry characterization was divided into surface chemistry (surface energy, surface functionalization, charge density along the length, etc.) and internal structure (degree of crystallinity, organization of amorphous chains, polymer chain morphology, etc.). Processing needs included measurements of aggregation and redispersion, processing-structure-property relationships for composites, and durability. Environmental health measurement needs were focused on concentration and particle detection in liquids and in air. These measurements are critical to enable the development of environmental health and safety standards for nanocellulose commercialization.

The participants representing the manufacturing sector agreed that the greatest measurement need is high-throughput characterization of CNM geometry (size and morphology), surface energy, and surface chemistry. Most of the current analytical techniques (electron microscopy, atomic force microscopy, etc.) used to quantify these key properties are too expensive (in terms of training, speed, and capital cost) for quality control in a manufacturing environment. Additionally, any new, faster techniques that are developed will still be required to maintain a sufficient level of sensitivity and resolution to detect changes in CNM properties during production.

The research and development discussion focused on three major commercial applications of CNM: nanocomposite systems, viscosity modifiers, and thin film barrier applications. To enable science-based advancement of these CNM applications, researchers were in agreement with the manufacturing sector, stating that the most critical measurement need is characterization of geometry and chemistry. These properties are essential for both manufacturing a consistent product and exploiting these properties for innovation within the application space. 
Finally, the standards breakout session identified a three-pronged approach to CNM commercialization. First, the development of standard test methods for key CNM properties (primarily morphology and chemistry) that enable consistency and quality control in manufacturing must be accomplished. Second, environmental health and safety (EH\&S) studies focused on CNM toxicity (for both manufacturing and commercial product release) as well as environmental impact prior to large-scale production should be conducted. Finally, reference materials, such as the wood-derived $\mathrm{CNC}$ powder (CNC-1) and $\mathrm{CNC}$ in aqueous suspension (CNCS-1) offered by NRC-Canada must be utilized to facilitate collaboration between academia and industry.

Currently, international efforts are underway in both the public and private sectors to develop new applications for CNM and to manufacture CNM as efficiently as possible. In parallel, manufacturing standards are also being developed by several organizations; within the ISO Technical Committee 22 and ASTM E-56. This workshop was a step towards understanding the measurement needs of this growing industrial sector and provided a path forward for ways in which NIST and other national measurement laboratories and standards organizations can focus their efforts and accelerate the sustainable commercialization of CNM. A key finding was that rapid and high-resolution size, morphology, and chemistry characterization tools must be designed and implemented to enable this exciting new material to be fully industrialized. 


\section{Introduction}

A one-day workshop focused on the Measurement Needs for Cellulosic Nanomaterials was organized by the National Institute of Standards and Technology and TAPPI on June 23, 2014. The goal of the workshop was to understand the measurement needs of this growing industrial sector and to begin to develop a path forward for NIST and other national measurement laboratories and standards organizations to help accelerate the commercialization.

Global investments in nanotechnology programs have led to a deeper appreciation of the high performance nature of cellulose nanomaterials and a first step in this process was publication and distribution of "Production and Applications of Cellulose Nanomaterials" by TAPPI in 2013. ${ }^{1}$ This book provided a high-level snapshot of the industry at that time and clearly demonstrated the global interest, the dynamic nature and multi-functionality of this new material, and the potential for new product development.

Cellulose, manufactured to the smallest possible size (roughly $2 \mathrm{~nm}$ x $100 \mathrm{~nm}$ ), has become a high-value material that enables products to be lighter and stronger, biologically compatible, and derived from readily renewable resources. ${ }^{2}$ In addition, there is a huge potential for a dramatic impact on the national economy. Cellulose-based nanotechnology creates a pathway for expanded and new markets utilizing these renewable materials. The installed capacity associated with the US pulp and paper industry represents an opportunity (with investment) to move rapidly to large-scale production of nano-based cellulosic materials. However, effective imaging, characterization and fundamental measurement science (metrology) for process control and characterization are currently lacking. This workshop was designed to initiate discussions of the needed measurements and the potential solutions.

Development of the characterization methods for advanced manufacturing of CNM is imperative to the success of this rising economic sector. This revolutionary technology will create new jobs and strengthen America's forest-based economy through industrial development and expansion. It allows this, previously perceived, low-tech industry to move directly into high-tech products and processes, and reverse its current economic decline. ${ }^{3,4}$

Nanocellulose has extremely small dimensions in both the radial and length direction. The development of appropriate nanoscale 3-D measurement methods and tools is a fundamental requirement. What are the correct measurements? What useful tools are currently available, and what still needs to be developed? What kind of documentary and physical standards are needed? These questions were posed to the participants of the workshop. Thus, this workshop was designed to open a dialog to evaluate current characterization methods, explore new technologies, and identify the most critical characterization and standardization needs. 


\section{List of Acronyms}

AFM - Atomic force microscopy

BET - Brunauer-Emmett-Teller surface analysis theory

CNC - Cellulose nanocrystal(s)

CNF - Cellulose nanofiber(s) (or nanofibril(s))

CNM - Cellulose (or cellulosic) nanomaterial(s)

CNT - Carbon nanotube

COD - Chemical oxygen demand

DIC -Differential interference contrast microscopy

DLS - Dynamic light scattering

DMA - Dynamic mechanical analysis

DP - Degree of polymerization

EH\&S - Environmental health and safety

FPL - Forest Products Laboratory

FRET - Förster (or fluorescence) resonance energy transfer

FT-IR - Fourier transform infrared spectroscopy

GHS - Globally Harmonized Standard

GRAS - Generally regarded as safe

HIM - Helium ion microscopy

ICP-OES - Inductively coupled plasma - optical emission spectroscopy

ISO - International Organization for Standardization

LCRA - Life cycle risk analysis

LSCM - Laser scanning confocal microscopy

NCC - Nanocrystalline cellulose

NFC - Nanofibrillated cellulose

NIOSH - National Institute for Occupational Safety and Health

NIST - National Institute of Standards and Technology

NMI - National Measurement Laboratory

NMR - Nuclear magnetic resonance spectroscopy

NRC-MSS - National Research Council - Measurement Science and Standards (Canada)

PPE - Personal protective equipment

OECD - Organisation for Economic Co-operation and Development

OSHA - Occupational Health and Safety Administration

REDOR - Rotational-echo double-resonance NMR

$\mathrm{RM}$ - Reference material(s)

RS - Raman spectroscopy

SEM - Scanning electron microscopy

STEM - Scanning tunneling electron microscopy

TAPPI - Technological Association of the Pulp and Paper Industry

TC - Technical committee (of ISO)

TEM - Transmission electron microscopy

TEMPO - (2,2,6,6-Tetramethylpiperidin-1-yl)oxy

TGA - Thermal gravimetric analysis

USDA - United States Department of Agriculture

UV-Vis - Ultraviolet / visible light spectroscopy

XPS - X-ray photoelectron spectroscopy

$\mathrm{XRD}$ - X-ray diffraction 


\section{Presentation Summaries}

\subsection{A New Biorefining Approach}

Kim Nelson, Vice President Nanocellulose Technology, American Process, Inc.

American Process Inc. is currently installing a nanocellulose demonstration line at the existing AVAP® Biorefinery in Thomaston, Georgia. This new production facility produces CNC, CNF, and hydrophobic, lignin-coated varieties of both directly from biomass. Key properties that this fractionation biorefinery process currently requires measurement of are hydrophobicity, crystallinity, transparency, thermal stability and purity. The most important limitation cited in this biorefining process is the cost effective preservation of discrete nanocellulose particle morphology during drying for effective re-dispersion in hydrophobic plastic resins. The lignincoated varieties appear to survive freeze-drying and spray-drying due to the screened hydrogen bonding interactions, enabling uniform re-dispersion of the powder into silicone and other hydrophobic polymers. There is a desire for a low cost method to eliminate hydrogen-bonding interactions in dried, bleached CNM that maintains the original surface properties, morphology and color (white).

Currently, the most important properties and corresponding characterization methods are:

- Particle size distribution (SEM, TEM)

- Lignin content (tedious wet chemistry)

- Crystallinity (XRD)

- Thermal stability (TGA)

- Purity (ICP-OES)
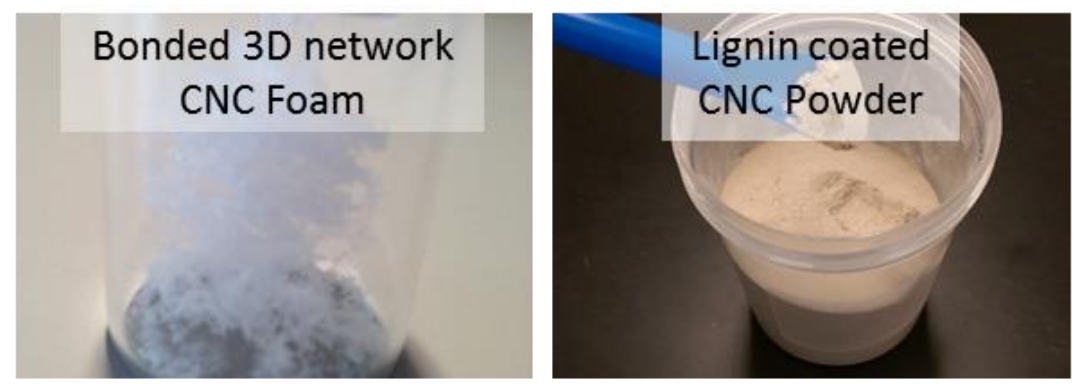

Freeze-Dried

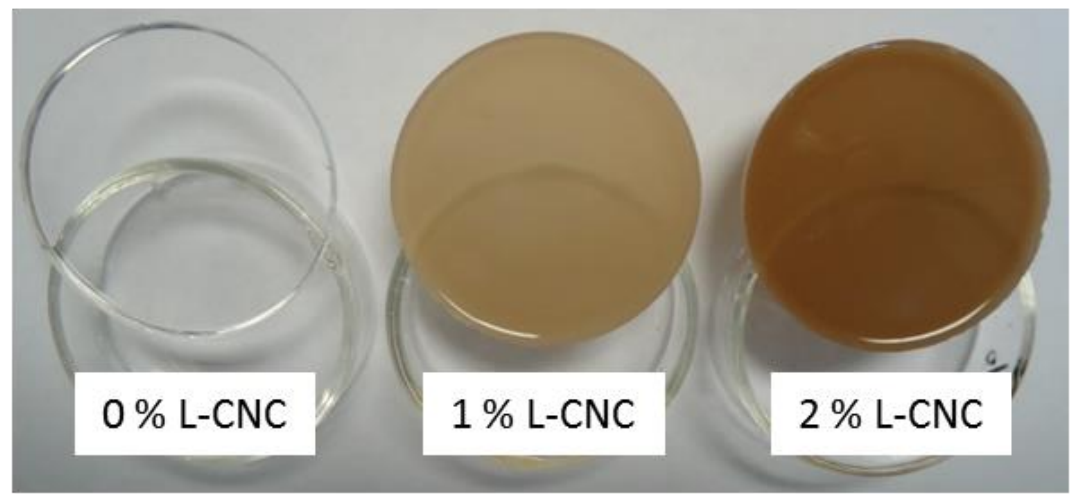

Lignin coated CNC/silicone composite

Figure 1: Lignin coated CNC is easily dispersed in hydrophobic materials. 
The limitations of the methods used to characterize each of these properties are cost, long sample preparation and characterization times, and the high level of technical expertise required to perform the measurements. Alternate methods that are less expensive (in both time and money) and require minimal training and expertise are needed to move cellulosic nanomaterial production forward.

\subsection{Real-Time Analysis of CNM during Production}

\section{Sean Ireland, Verso}

For full-scale production of cellulose nanomaterials, several key measurement issues remain that complicate and hinder advancement of the industry. Manufacturing CNM is energy intensive, expensive (in terms of both time and money), can involve hazardous chemicals, and requires the incorporation of large production volumes. Currently, there is no "real-time" analysis method available for process control at the production rates and volumes necessary to make CNM a profitable, industrially relevant material. Batch sampling, including sample preparation and measurement (whether it is rheology and/or electron microscopy), assumes that a $1 \mathrm{~L}$ sample is representative of 10 to $50 \mathrm{~kL}$ of material and typically requires an hour or more for results. From a high throughput production perspective, this cycle is too slow.

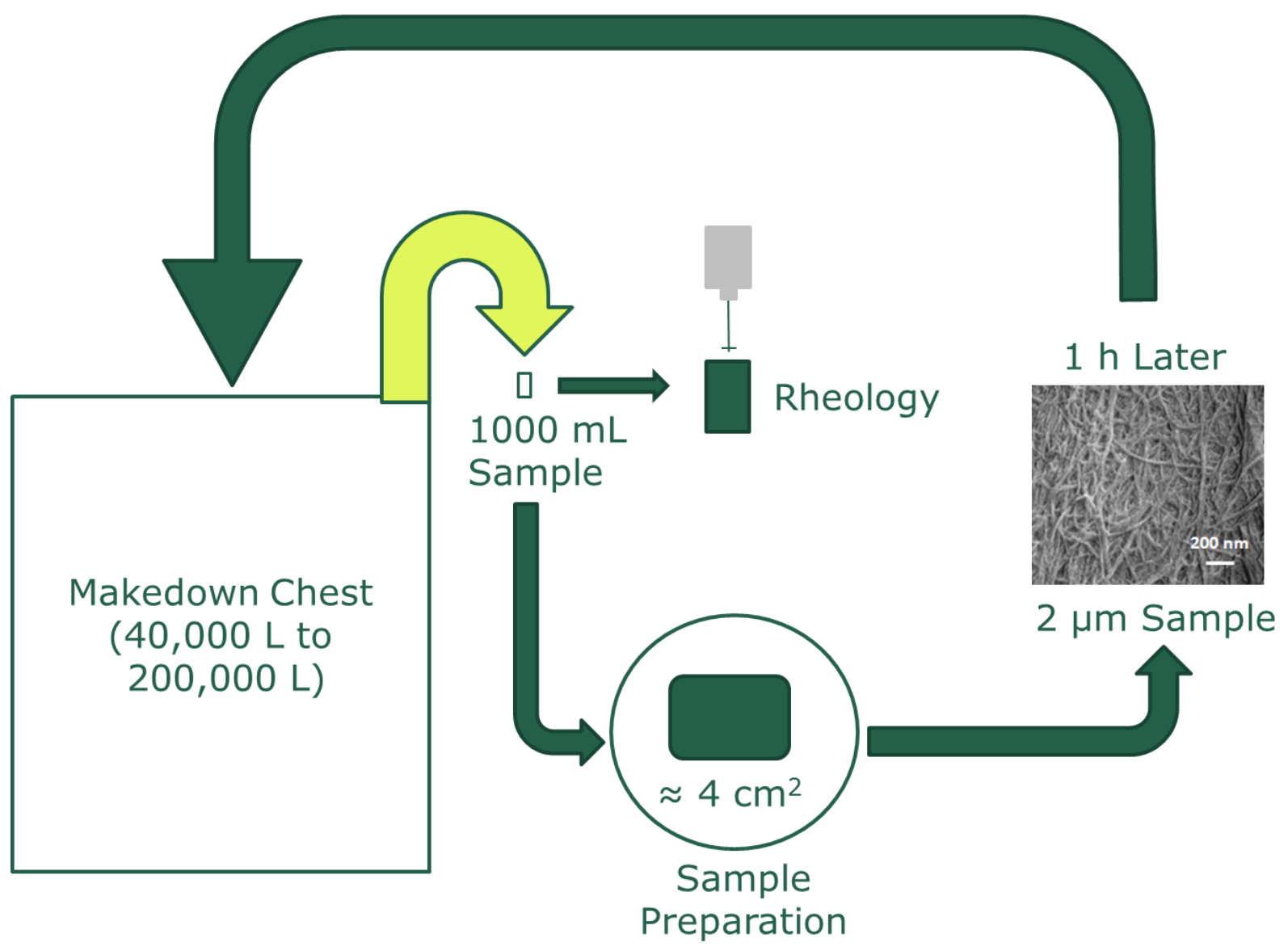

Figure 2: Current quality control measurement limitations

Current measurement options are SEM, TEM, AFM, XRD, XPS, UV-Vis, and Rheology. These techniques provide the answers that industry requires for efficient processing of uniform 
material, but they are slow. The optimal measurement technique would be fast, low cost, online (required to obtain an estimate of specific physical properties), and precise (accurate and reliable as well.) Potential key measurements that are desired for industrial scale up are both size (length and aspect ratio) and crystallinity.

\subsection{A Pilot Producer's Perspective}

\section{Alan Rudie, Forest Products Laboratory}

The Forest Products Laboratory (FPL) pilot scale CNC and CNF (TEMPO grade) production facility is located in Maine and provides materials sold through the University of Maine's Process Development Center. The CNC are produced from commercially available dissolving pulps in $25 \mathrm{~kg}$ batches through a $64 \%$ sulfuric acid procedure. The CNF are prepared in $2 \mathrm{~kg}$ batches from bleached pulp by the TEMPO pretreatment process developed by Isogai. ${ }^{20,21} \mathrm{~A}$ critical step required for industrial scale production of CNC and CNF is removing water and liquid from the material to enable shipment of the cellulosic material in a dry form. Freezedrying is an effective method. FPL utilizes an ice slushy machine and a butyl alcohol co-solvent to accomplish the initial drying and then completes the drying process in a $150 \mathrm{~L}$ freeze drier. Following this process, CNC can be easily re-suspended in water with no obvious degradation in the suspension quality. However, TEMPO CNF is difficult to re-suspend after this freeze-drying process.

Currently, the FPL pilot production facility measures the following parameters for cellulosic nanomaterials:

- $\mathrm{CNC}$ :

○ sulfur content (ICP-OES),

○ \% suspension solids (chemical oxygen demand, COD),

○ suspension conductivity

○ Crystallinity (occasionally)

- CNF:

acid group content (titration),

○ $\%$ suspension solids (COD),

- suspension visual clarity

Several key properties that are difficult to quantify but are desired measurements for FPL are:

- $\mathrm{CNC}$

- Particle diameter

- Aggregation

- Particle length

○ DP

- $\mathrm{CNF}$

○ Particle diameter

- Branching

- Particle length

○ DP

○ Rheology

Of these properties that FPL would like to quantify, several (particle diameter and length, aggregation, branching, etc.) could be readily observed through EM or AFM methods. However, 
these techniques are expensive and are not currently designed for high throughput/quality control applications. The time and cost of performing microscopy on each batch is prohibitive. Additionally, concerns have been raised that sample preparation for microscopy could potentially introduce variation from batch to batch. Laser light scattering would allow for higher throughput, in line monitoring of the material but the most critical dimension (particle diameter) is outside of the quantifiable length scale of this technique.

A health and safety metrology concern raised by FPL is that of cellulosic nanomaterial particulate dispersion in air. Some existing techniques rely on light scattering but it is possible that some CNM particulate matter is too small to be detected. Other methods observe adsorbed particulates on a filter and FPL has had some success with ion exchanging Cesium to allow for detection. While effective on the lab scale, labeling production quantities with expensive materials is not practical. Environmental health and safety concerns are further exacerbated by variables such as acid functional group distribution (along the length of the CNC) and surface chemistry variation.

\subsection{CNF Pilot Plant Overview}

\section{Michael Bilodeau, University of Maine}

The University of Maine CNF Pilot facility consists of a CNF refiner that allows pre-treatment of wood pulp and a mass colloider grinder. The facility is capable of producing up to one ton of CNF slurry (3 mass \% solids) per day and is available on a fee-for-service basis. Currently, a Techpap MorFi Fiber Analyzer is used to monitor the process and perform rough CNF characterization. The device provides morphological information regarding fiber length and width. It also has a quick turnaround and high reproducibility. It is capable of characterizing micrometer size particles but does not measure submicrometer lengths.

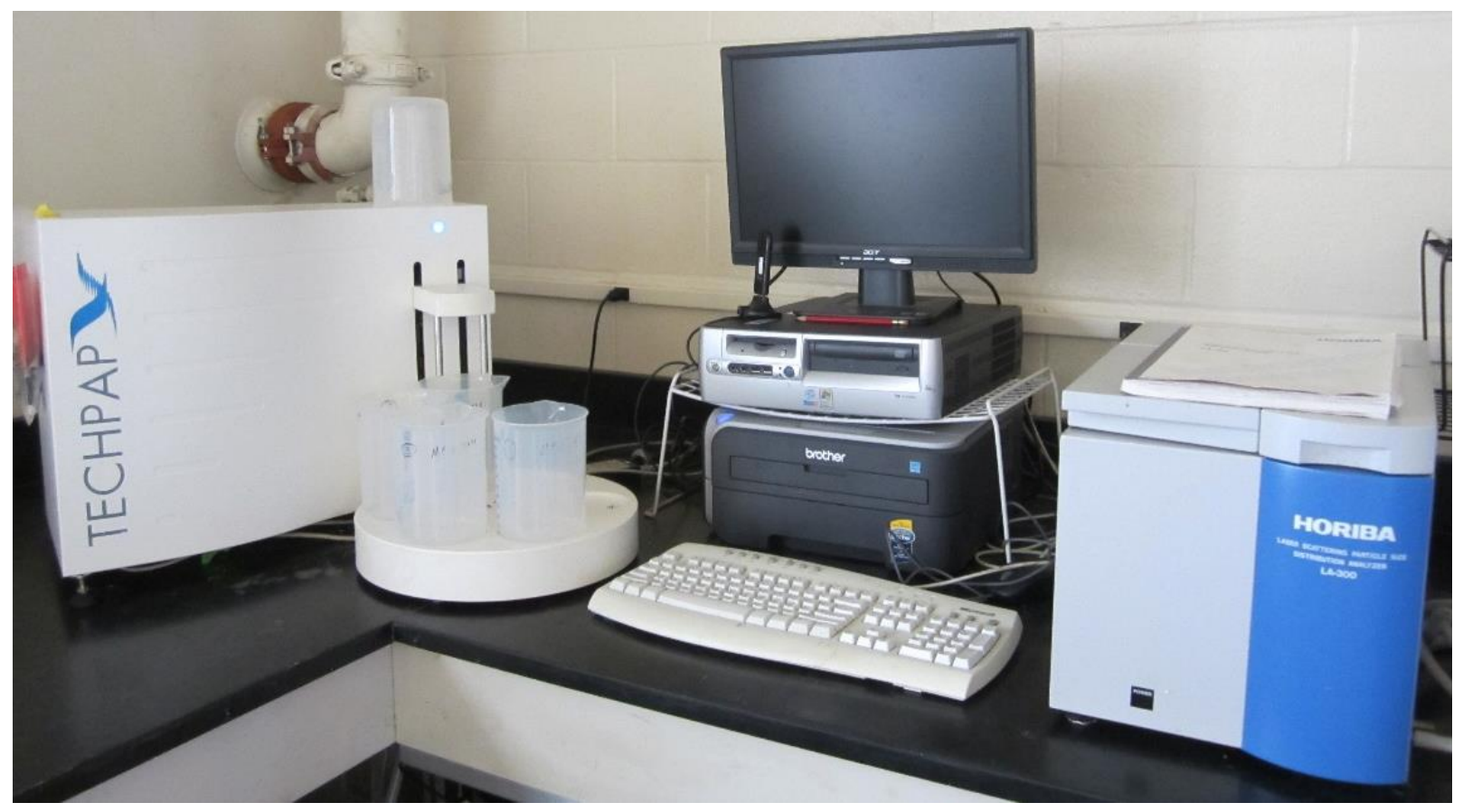

Figure 3: TechPap MorFi Fiber Analyzer at CNF Pilot Plant 
For the purposes of the University of Maine CNF Pilot facility, the ideal set of CNF measurement tools would meet these requirements:

- reproducible,

- on-line,

- complete in minutes, and

- in good agreement with off-line quality control tests.

Additionally, less critical but still of interest are the measurement of viscosity, water holding capacity, surface area, and surface charge. A device capable of performing these measurements needs to be developed to allow for effective process control during the production of CNF.

\subsection{An Academic's Perspective}

Robert J. Moon, Forest Products Laboratory, US Forest Service, Georgia Institute of Technology \& Purdue University

Focusing more on the fundamental scientific aspects of cellulosic nanomaterials, work has been performed to characterize the mechanical properties of individual nanocellulose particles and composite systems containing CNM. Employing innovative AFM mechanical measurements, the modulus and surface energy along the long axis of a single CNC can be observed. The thermal expansion of shear-oriented and self-organized films of CNC have also been explored. ${ }^{18} \mathrm{CNC}$ have been shown to improve the flexural strength of cement composites at low $(<1.5$ vol\%) loadings.

In the field of electron microscopy observation of CNM, several key measurement needs were identified. One concern was the loss of sharpness and edge retention due to E-beam ablation. Also, when organic CNM is observed in a matrix that also contains inorganic particles, contrast between the organic matrix and the organic CNM is particularly difficult. ${ }^{19}$ The addition of conductive coatings that are traditionally used to minimize beam damage in electron microscopy add a few nanometers of material to the sample, blurring the sharp features of the CNM. A major improvement in EM characterization technology would be the ability to obtain images of CNM without the application of a conductive coating to the sample.

Key CNM properties that need to be measured are:

- Morphology and surface chemistry

Oniformity

- Identification of CNM exposed surfaces

- Location of chemical reactive sites on CNM surface

- Morphology (stepped surface contours, agglomeration, branching)

- Self-assembly (chiral, network)

- Crystallinity with CNM

○ Chain arrangement \& location of crystalline vs disordered regions

- Paracrystallinity

- Surface vs. bulk characterization

- Identify necessary input parameters for predictive modeling

- Thermal and mechanical properties

- Property anisotropy

- Uniformity of properties along CNM length

Interfaces (CNM-CNM, CNM-matrix, etc) 
- Load transfer between CNM and matrix

$\circ$ Interaction of CNM with the surrounding environment

- $\quad$ CNM shelf life

How do we test shelf life?

- How do we quantify?

Overall, the measurement needs for several specific techniques and applications are:

- $\mathrm{AFM}$ of CNMs

○ Quantitative measurements (mechanical, surface energy \& chemistry)

- Uniformity of properties along CNM length (property mapping)

Interfaces (CNM-CNM, CNM-matrix)

- Functionalization

- Identify location

$\circ$ Bonding strength

- See both inorganic and organic at same time

- CNM-Composites

- Dispersion of CNM within matrix

Interface (CNM-CNM, CNM-matrix)

○ Pull out test

- Continuous Fibers

- In situ $\mathrm{CNM}$ flow orientation

- Microfluidics

○ In situ self-assembly

- Predictive Modeling

- Input parameters

- Atomic and nano scale

- Flexible Electronics

- Surface roughness

○ Thermal properties

- Cements

○ Locating CNMs within the composite structure

\subsection{Adaptive CNC Composites}

"Mechanically Adaptive Materials Reinforced with Tunicin-Nanocellulose"

\section{E. Johan Foster, Adolphe Merkle Institute and Virginia Tech}

The development of adaptive materials based on $\mathrm{CNC}$ has the potential to provide a large industrial market for nanocellulosic materials. The addition of light responsive molecules such as benzophenone, ${ }^{7}$ coumarin, ${ }^{8}$ and ureidopyrimidone ${ }^{9}$ to the cellulose backbone allow permanent or $\mathrm{r}$ eversible crosslinking between $\mathrm{CNC}$ and the surrounding matrix within nanocomposite systems. 


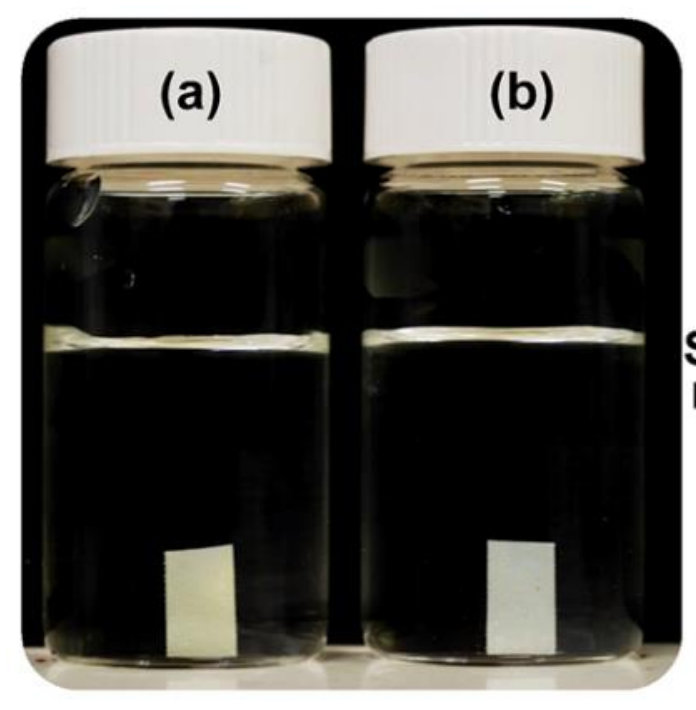

UV irradiated
Not irradiated

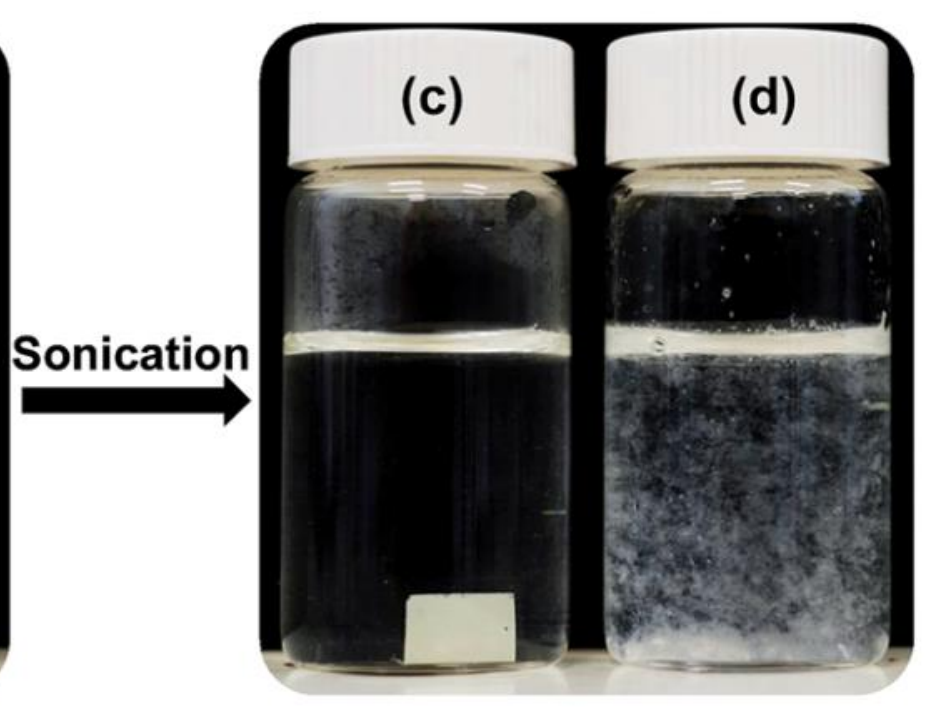

UV irradiated Not irradiated

Figure 4: CNC films which have been permanently crosslinked (or not) by UV irradiation. ${ }^{7}$

Smart design can provide the next generation of materials. Moderating non-covalent and covalent interactions between small molecules, particles, telechelic building blocks, or combinations thereof by external stimuli is a powerful and simple approach to create useful new materials with adaptive properties. CNC provide a fantastic opportunity to reinforce and add smart aspects to polymeric systems. Synthetic protocols have been developed to process a wide variety of materials.

Critical measurement needs to enable applications of CNC in adaptive materials are the functionalization amount or coverage of a particular chemical moiety, size (aspect ratio), and charge density. A particular question raised by this work is what causes tunicate derived CNC to perform "better" than cotton CNC in reinforcement applications.

\subsection{Solid-State Characteristics of $\mathrm{CNC}$}

\section{Wadood Y. Hamad, Principal Scientist, FPInnovations}

The structure of the crystalline portions of cellulose has been studied extensively with, for example, X-ray diffraction, Fourier transform infrared spectroscopy (FT-IR), electron diffraction, neutron diffraction, as well as solid-state nuclear magnetic resonance (NMR) spectroscopy. VanderHart and Atalla ${ }^{10}$ conclusively showed using ${ }^{13} \mathrm{C}$ cross-polarization magic angle spinning (CP/MAS) NMR that native cellulose's crystalline regions contain a mixture of two different structures, known as cellulose $\mathrm{I}_{\alpha}$ and cellulose $\mathrm{I}_{\beta}$, which differ in both the conformation of the glucosidic linkages and hydroxymethyl groups as well as the location of hydrogen bonds connecting adjacent strands. Signals from these along with surface, disordered, and paracrystalline regions simultaneously contribute to the NMR spectra. Wickholm et al. ${ }^{11}$ have assigned separate $I_{\alpha}$ and $I_{\beta}$ crystallites in addition to paracrystalline cellulose and three different surface peaks for $\mathrm{C}_{4}$ in high-resolution birch and cotton cellulose spectra. It has been suggested that after acid hydrolysis, only the highly crystalline rod-like regions remain ${ }^{12}$, and in view of the findings of Wickholm et al., ${ }^{11}$ the crystallinity obtained from X-ray diffraction of cellulose nanocrystals likely includes both crystalline and paracrystalline regions. NMR spectra of cellulose nanocrystals have actually been shown to contain surface/amorphous peaks that 
appear to be much larger than would be expected from a material with a high degree $(>80 \%)$ of crystallinity ${ }^{13}$.

NMR and X-ray diffraction analyses ${ }^{14}$ suggest that CNC consist of a broad distribution of environments having varying crystalline perfection and dynamics. The vast majority of any particular CNC particle is well structured to diffract X-rays, but because well-ordered material should exhibit the crystalline chemical shifts and be protected from ${ }^{1} \mathrm{H} /{ }^{2} \mathrm{H}$ exchange, at most about $40 \%$ of any acid-hydrolyzed CNC can be thought of as highly or well-crystallized. The remainder shows varying degrees of disorder. Moreover, acid-hydrolyzed CNC consists not of sharply defined exchanged and nonexchanged regions as might be expected for highly crystalline, high-surface area particles, rather they are more uniformly partially exchanged. Within the exchanged regions however, the three exchangeable sites appear to be unevenly exchanged, with $\mathrm{H}_{6}$ exchanging more readily than $\mathrm{H}_{2}$ or $\mathrm{H}_{3}$.

While the $\mathrm{C}_{4}$, is assigned to less well-structured cellulose chains-expected in amorphous and surface regions, the fact that the $\mathrm{C}_{4}$ peak is also significantly dephased in the rotational-echo double-resonance (REDOR) NMR experiments suggests significant exchange into more crystalline material as well. These results indicate that rather than well-defined crystalline and non-crystalline regions, CNC contains a more finely varied distribution of environments. In particular, the incomplete exchange at the amorphous $\mathrm{C}_{4}$, site strongly suggests that acidhydrolyzed CNC particles contain abundant disordered domains in their interiors, protected from exchange by enveloping crystalline regions. Recently, experimental evidence indicates the ability to effectively use Raman spectroscopy, along with optical techniques, to monitor defects in CNC structure $^{15}$.

Although NMR spectroscopy techniques typically require long testing times and a high degree of analysis, it has been proposed that alternate forms of spectroscopy such as fluorescence and Raman could be used to monitor the formation of crystal defects and impurities during processing. Knowledge gained through detailed NMR studies of CNC crystalline structure will inform the development of these in-line process monitoring metrology techniques.

\subsection{The Standards Community's Perspective}

\section{Linda J. Johnston, Measurement Science and Standards, NRC Canada}

Canada's National Metrology Institute (NRC-MSS) is developing cellulose nanocrystal reference materials. The organization is also working on the ISO Technical Committee 229 (TC 229) to deliver a technical report on CNC characterization. The Canadian Standards Association is also developing CSA Z5100 (Cellulosic Nanomaterials Test Methods for Characterization). These efforts are driven by in-house research and development that strives to develop standard protocols for measuring size distributions, the dispersion of $\mathrm{CNC}$ in composite materials, and CNC particle concentration in solution.

Reference Materials (RM) are needed for calibration purposes, development and validation of protocols, environmental health and safety testing, and standards and regulations. The NRC-MSS released two CNC RM in 2013: (1) CNC prepared by sulfuric acid hydrolysis of wood pulp by FPInnovations: 6 mass\% Aqueous Suspension (CNCS-1) and (2) Dry Powder (CNC-1). ${ }^{16}$ These materials were characterized in-house and by collaborators. The reference materials are shipped with certified properties and informational data. Measurements conducted to certify these standard materials include: 
- $\quad$ Particle morphology (AFM, SEM, TEM, DLS)

- Crystallinity (XRD, NMR)

- Surface characterization (Raman, FTIR, zeta potential)

- $\quad$ Surface area (Brunauer-Emmett-Teller Approach, BET) ${ }^{17}$

- Composition (conductometric titration, ICP-OES, ICP-MS)

- Density

- Thermal stability (TGA)

Several challenges have complicated the production of additional reference materials. These challenges include 1) the absence of standard protocols for sample preparation, measurements, data analysis; 2) the identification of key measurements for an inter-laboratory comparison with larger data sets for individual measurements; and 3) the consideration of requirements for certified (metrologically validated method and traceability, uncertainty analysis) vs. reference vs. informational RMs.

As mentioned above, the ISO TC 229: Technical Report 19716: Nanotechnologies Characterization of Cellulose Nanocrystals: Particle Morphology, Purity and Surface Properties is currently under development. For the scope and contents addressed in this report, see Appendix 10.3. The status of the project is as follows:

- Project approved in April 2014

- Expert team in place (14 experts from 7 countries)

- First project meeting in May 2014 at ISO TC 229 interim meeting of Measurement and Characterization Working Group

- Liaison with TC6 - Paper, Board and Pulp. Recently expanded scope to cover cellulosic nanomaterials, including terminology, sampling procedures, test methods, product and quality specifications, and the establishment and maintenance of appropriate calibration systems

- Task Group on Cellulosic Nanomaterials with Dr. Jean Bouchard (Canada) as the facilitator and Dr. Colleen Walker (USA) as the secretary

- Goal: Identify ISO projects (TR, TS, standards) on CNMs and advise on which WG shall lead; examine the possibility of including CNM specificity factors in existing TC6 standards when they come up for systematic review.

- $\quad$ Liaison with TCs on EM, surface analysis, particle counting

Several measurement challenges and opportunities have been identified which include:

1. Morphology - particle size, shape, size distributions - correlate ensemble methods such as DLS with particle counting methods (EM, AFM) - number count distributions vs volume or intensity weighted distributions (EU nanomaterial definition)- dispersion, aggregation, agglomeration - broad size distributions of nanorods- correlation of size with properties. Modified CNC - what measurement is needed?

2. Surface Area - Conventional BET problematic for dry CNC

3. Crystallinity - Different methods, different values

4. Composition - What are key measurements?

5. Surface properties, chemistry - Key to controlling solution behavior, surface modification and compatibilization with composite materials 
Table 1: Measurement challenges: Value chain for CNM characterization and measurement. Adapted from CSA Z5100 Cellulosic Nanomaterials: Test Methods for Characterization

\begin{tabular}{|c|c|c|c|c|}
\hline \multicolumn{2}{|c|}{$\begin{array}{l}\text { Base cellulosic } \\
\text { nanomaterials }\end{array}$} & \multicolumn{3}{|c|}{$\begin{array}{c}\text { Cellulosic nanomaterial enhanced or } \\
\text { enabled products }\end{array}$} \\
\hline $\begin{array}{c}\text { Cellulosic } \\
\text { Nanomaterial }\end{array}$ & Characterization & $\begin{array}{c}\text { Modification \& } \\
\text { Compatibilization }\end{array}$ & Dispersion & $\begin{array}{c}\text { Inclusion in } \\
\text { Nano-Enhanced } \\
\text { Products } \\
\end{array}$ \\
\hline $\begin{array}{l}\text { Cellulose } \\
\text { Nanocrystals } \\
\text { (CNC) } \\
\text { Cellulose } \\
\text { Nanofibrils } \\
\text { (CNF) }\end{array}$ & $\begin{array}{l}\text { Purity } \\
\text { Size } \\
\text { Physical } \\
\text { properties } \\
\text { Chemical } \\
\text { properties } \\
\text { Dispersibility in } \\
\text { water }\end{array}$ & $\begin{array}{l}\text { Surface } \\
\text { Modification } \\
\text { Surface } \\
\text { Compatibiliza- } \\
\text { tion }\end{array}$ & $\begin{array}{l}\text { Processes } \\
\text { Conditions } \\
\text { Dispersion } \\
\text { level in matrix }\end{array}$ & $\begin{array}{c}\text { Degree of } \\
\text { enhancement of } \\
\text { target } \\
\text { properties } \\
\text { (per level of } \\
\text { cellulosic } \\
\text { nanomaterial) }\end{array}$ \\
\hline & $\begin{array}{r}\text { Envirc } \\
\text { Con } \\
\text { Comm }\end{array}$ & $\begin{array}{l}\text { cial Product Requi } \\
\text { Sustainability }\end{array}$ & $\begin{array}{l}\text { (EHS) } \\
\text { cle } \\
\text { nents }\end{array}$ & \\
\hline
\end{tabular}

\subsection{Service Life Prediction for CNM Composites}

Aaron M. Forster, Engineering Laboratory, National Institute of Standards and Technology

The ability to accurately predict the service life of new materials is critical to provide confidence for market acceptance of new technologies from the bench to commercialization. Successful prediction requires accurate characterization metrologies, scientifically grounded accelerating environments, and accurate physical or statistical models of performance changes over time. Currently, the characterization measurement science needs are:

- Mechanical and transient properties

- Fracture properties

- Thermal properties

- Moisture diffusion and water content

The service life prediction models developed at NIST account for key external stressors: temperature, humidity, mechanical fatigue, and UV exposure ${ }^{5}$ and have been applied to coatings, sealants, and nanocomposites. 


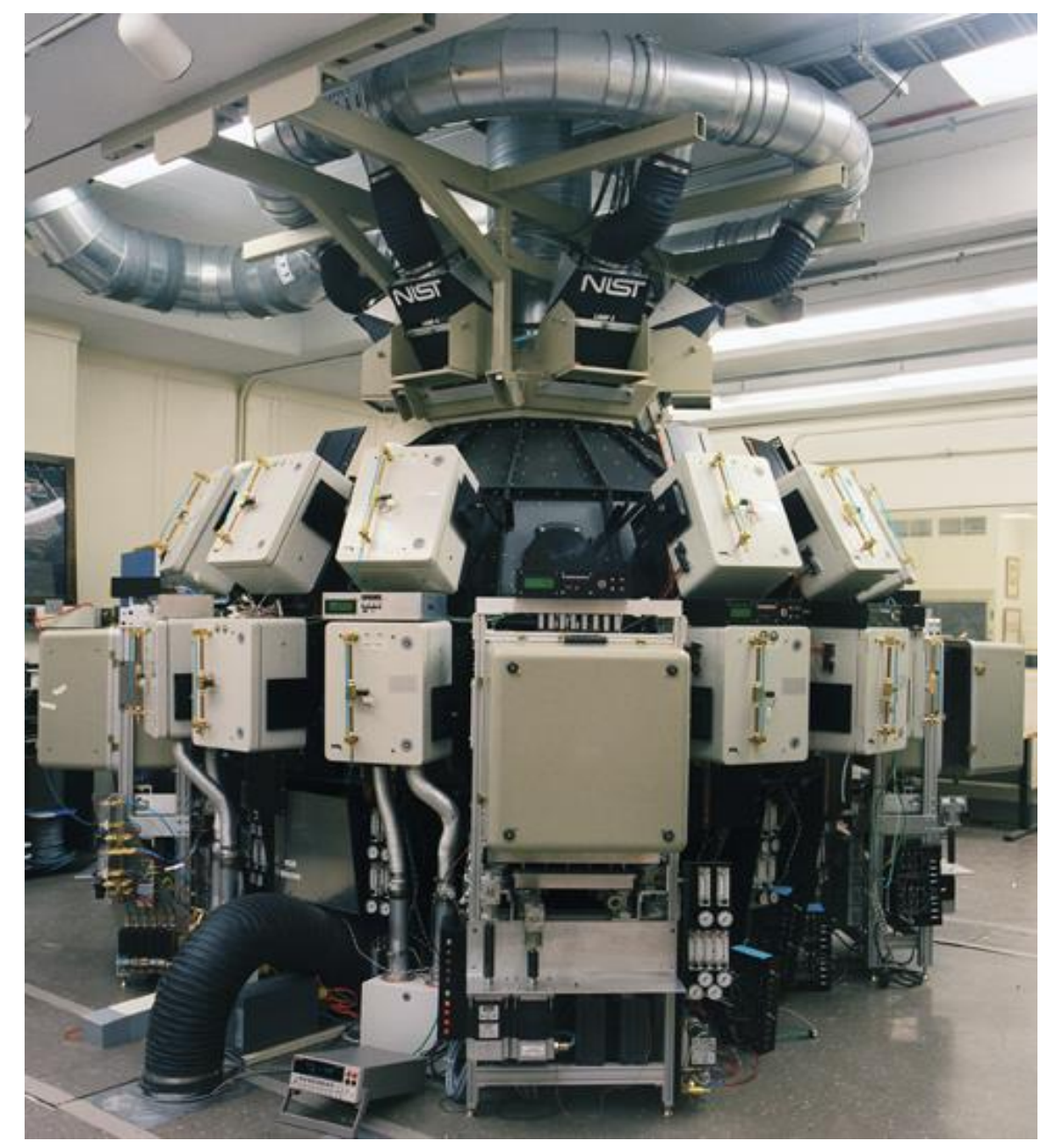

Figure 5: Integrating Sphere utilized for accelerated weathering of materials to enable service life prediction. ${ }^{6}$

The long-term properties of CNM-containing nanocomposites have not been fully evaluated to allow direct comparison of this new technology with traditional technologies. A critical consideration that is of particular importance in hydrophilic, biologically derived materials (such as nanocellulose) is the impact of moisture on filler and composite properties including:

- Crystallinity

- Disruption of percolated networks

- Accessibility of hydroxyl groups

- Chemical functionalization of the fillers

The current challenges associated with measuring the service life of CNM are (1) understanding the long-term impact of moisture and UV on nanocellulose as a function of internal structure and surface chemistry and (2) determining the standard exposure and measurement methods required to enable reliable service life prediction. Potential limitations or key factors to consider as research techniques are developed to address these challenges are the distribution of geometries found in natural materials, achieving an adequate level of dispersion of the CNM, and quantifying the surface chemistry of the CNM. 


\subsection{CNM Environmental Health and Safety Roadmap}

\section{Contributed by Jo Anne Shatkin, Ted Wegner and Kathleen Bennett}

The nanoscale characteristics of cellulose nanomaterials (CNM) require an assessment of Environmental Health and Safety (EH\&S) to ensure that materials and products can be manufactured and used without negative impacts. Assessment of EH\&S at the nanoscale presents challenges due to the difficulty of detecting and accurately measuring small particles. The fibrous nature of CNM and its organic disposition complicate this challenge. However, it is critical to commercial success to adequately demonstrate the safety of CNM in terms of physical and chemical properties, human health and the environment. This presents a challenge due to the need for different measurement techniques.

The present version of the EH\&S roadmap for cellulose nanomaterials is described in more detail in Appendix 10.4. Identified below are data gaps and priorities for both CNC and CNF.

\section{Safe Exposure Levels}

Currently there are no established and vetted occupational exposure limits specific to cellulose nanomaterials and engineered cellulose nanomaterials. Preliminary data indicate that cellulose nanomaterials are bio-durable in artificial lung fluid even after one year of exposure. In addition, preliminary in vitro studies have indicated that these materials can cause both inflammation and hypertrophy. Based on current information, the primary potential hazard for an occupational user currently associated with cellulose nanomaterials is the inhalation of pure, dried material during laboratory research and development and commercial production and manufacturing.

The potential hazards identified by the National Institute for Occupational Safety and Health (NIOSH) at each life cycle stage are listed in Table 2. 
Table 2: Hazards by Life Cycle and Application

\begin{tabular}{|c|c|c|}
\hline Life Cycle Stage & $\begin{array}{l}\text { Level of Certainty re: } \\
\text { Potential Hazards }\end{array}$ & Potential Hazards \\
\hline $\begin{array}{l}\text { Cellulose } \\
\text { Nanomaterials } \\
\text { Production }\end{array}$ & $\begin{array}{l}\text { Greater certainty of activities } \\
\text { due to controlled production } \\
\text { facility environment }\end{array}$ & $\begin{array}{l}\text { - Spills while physically moving both wet and } \\
\text { dried material } \\
\text { - Bagging/packing dried material for shipment } \\
\text { - Dried material into hood ventilation } \\
\text { - Dried materials washed into sewer system } \\
\text { - Wet material wasted into sewer system }\end{array}$ \\
\hline $\begin{array}{l}\text { Product } \\
\text { Manufacture }\end{array}$ & $\begin{array}{l}\text { Less certainty due to lack of } \\
\text { detailed knowledge of the } \\
\text { product manufacturing } \\
\text { practices of the various } \\
\text { facilities experimenting with } \\
\text { CNC-containing product } \\
\text { development (R\&D and } \\
\text { manufacturing activities) }\end{array}$ & $\begin{array}{l}\text { - Storage of dried material leading to } \\
\text { explosive conditions } \\
\text { - Opening shipment of dried material } \\
\text { - Mixing dried material with other materials to } \\
\text { create new product } \\
\text { - Dried material into hood/facility ventilation } \\
\text { - Machining or sanding dried material } \\
\text { - Spills and wet material wasted into sewer } \\
\text { system }\end{array}$ \\
\hline Consumer Use & $\begin{array}{l}\text { Low level of certainty around } \\
\text { use due to R\&D stage of most } \\
\text { products (exact product } \\
\text { makeup and how } \\
\text { manufacturers test product } \\
\text { performance) }\end{array}$ & $\begin{array}{l}\text { - Products breaking or degrading and } \\
\text { releasing cellulose nanomaterials } \\
\text { - Physical/chemical changes to product due to } \\
\text { unintended uses (e.g., composting packaging } \\
\text { for gardening) }\end{array}$ \\
\hline Post Use & $\begin{array}{l}\text { Low level of certainty around } \\
\text { durability and recyclability }\end{array}$ & $\begin{array}{l}\text { - Product degradation and potential release } \\
\text { (cellulose nanomaterials degrade, but may } \\
\text { be functionalized) } \\
\text { - Unintended uses and pathways }\end{array}$ \\
\hline $\begin{array}{l}\text { Product End-of- } \\
\text { Life }\end{array}$ & $\begin{array}{l}\text { Low level of certainty around } \\
\text { how products will decay in } \\
\text { different environments and } \\
\text { under different conditions, } \\
\text { and whether product decay is } \\
\text { "biodegradable" process }\end{array}$ & $\begin{array}{l}\text { - Degradation in landfill (controlled } \\
\text { environment) } \\
\text { - Degradation in uncontrolled environment } \\
\text { (e.g., littered) }\end{array}$ \\
\hline
\end{tabular}

\section{Measurement}

Measurement needs are prioritized in the EH\&S roadmap because, without them, none of the other needed studies can be conducted in a reliable way. This includes confirmatory detection techniques, quantitative measurements, and standardized protocols for sampling and analysis in environmental media, including air, water, and solid matrices.

\section{Physical-Chemical Data}

The assessment of safety for nanomaterials requires better characterization of key properties that may influence biological and environmental behavior. These measurements are not required only 
for dry and aqueous solutions, but also for as-delivered forms in toxicology experiments to measure dose.

\section{Human Health}

A variety of existing short-term (acute) toxicity test methods for testing chemicals was used to assess CNC and CNF, as were some novel assays. Most of these were in vitro assays. However, it is not clear whether any of the assays are appropriate for testing nanomaterials generally, or CNM specifically. There is a need to confirm the methods work for the stated purpose, as some standard toxicity tests have been shown to give false results due to interference of the nanoparticles with test reagents or media. The Organisation for Economic Co-operation and Development (OECD) has been evaluating its standard methods for applicability to nanomaterials. These methods need to be reviewed and considered in terms of their ability to report on the safety of CNM.

\section{Bridging Studies}

Bridging studies that link existing whole animal (in vivo) studies to in vitro tests are an opportunity to further develop in vitro methods for future assessments. First, there is a need for in vivo testing of $\mathrm{CNC}$ and $\mathrm{CNF}$ to evaluate whether they behave similarly to other forms of cellulose that are currently used in food and medical applications.

Current evidence is inadequate for this demonstration. Oral exposure studies are considered high priority because 1) several forms of cellulose are already approved by FDA and others for use as food additives and food contact substances, or are grandfathered as Generally Regarded as Safe (GRAS); 2) assessing whether novel nano-forms behave differently from approved forms has potential to open food-contact applications; 3) this work is prioritized because further animal testing will not be required if differences in uptake are not observed; 4) a sound comparison of in vitro tests with in vivo (whole animal) bioassays will create an easier path forward for surfacefunctionalized and materials produced by alternate pathways to be assessed for safety. It will allow for bridging studies, so that animal testing need not be repeated for each surface modification.

\section{Inhalation}

Inhalation exposure studies are needed because all foreign particles are potentially hazardous to the lung, and there are no nano-specific requirements for workplace safety. There is a need to assess the effects of inhalation exposure in the workplace. Further, additional endpoints should be studied to demonstrate overall safety in a breadth of uses. Long-term exposure studies are needed. To date, no studies exist that have explored the following endpoints in animal systems: neurotoxicity, carcinogenicity and reproductive/developmental toxicity.

\section{Environmental Safety}

While CNM is presumed to be a low hazard to the environment, studies are needed to demonstrate this. Environmental claims must be substantiated with data to demonstrate the safety and sustainability not only of the CNM, but also of its production process and use in products. Doing so would facilitate entry into more markets based on environmental performance. 


\section{Breakout Session Reports}

Breakout sessions were held in the afternoon to allow small group discussions of CNM measurement requirements specific to three sectors: manufacturing, research and development, and standards. Each community focused primarily on the most pressing measurement needs specific to its area. While many critical characterization techniques and CNM properties were of concern to each breakout group, key differences quickly became apparent. Each of the three breakout sessions discussed the importance of the quantification of morphology (particularly aspect ratio) and surface properties (sulfate ester content and surface energy). Discussions from the manufacturing breakout session also focused on the development of high-throughput, economical characterization processes. Research and development professionals called for the development of modeling tools and high resolution, high contrast imaging methods. The standards development session revealed a need for particle detection in both liquid and gaseous dispersions. Summaries of all three discussions follow.

\subsection{Manufacturing Breakout Session Breakout Session Summary}

\section{Co-Chairs: Alan Rudie and Michael Postek}

The manufacturing breakout session focused on the metrology requirements for CNM that will enable large-scale industrial production. Discussions touched on the high throughput measurements required for quality control and in-line processing. The manufacturing breakout session discussion was driven by several key questions posed by the organizers. Each of these questions were addressed and discussed at length. The results have been summarized below:

What measurements need to be made to assure that the preparation of the CNM meets the projected target for quality control?

The quality control and analysis (QC and QA, respectively) of incoming material (kraft pulp or biomass like woodchips/agricultural residues) is thought to be largely under control due to the established nature of the pulp and paper industry. However, there are still many outstanding questions regarding the QC/QA of outgoing CNM. Currently, several measurements are performed during CNM production. These measurements include DLS, OES, DIC, FT-IR, UVVis (see definitions in Tables 3 and 4 below). (DIC is a particle size measurement which uses a flow-through device to determine particle length and width. The University of Maine Pilot Plant uses DIC to detect particles down to $t<0.1 \mathrm{~mm}$ at which point refining is considered complete.)

Several desired QC measurements and ideas for future production metrology tools included: (1) a DIC device using short wavelength light (X-ray) to determine particle size (allowing smaller particle detection), and (2) an NMR procedure to determine surface area. Additionally, inline shape/size measurements are needed for CNC production. These parameters dictate surface area and most other properties and there is currently no fast method available to quantify CNC geometry.

A few other CNM properties and measurement concerns were mentioned other than surface properties and CNM geometry. Specifically, branching is an important consideration for_CNF. There are little data available on the determination of branching. Mechanically derived CNF is thought to be branched while the morphological structure of chemically produced CNF is unknown. In addition, although health and safety concerns are considered more thoroughly in the Standards Breakout Session Summary, EH\&S concerns are critically important in the manufacturing of CNM. Specific concerns were raised regarding the validation of existing air 
monitoring equipment for CNM detection to prevent inadvertent exposure to air-dispersed particles during production.

What (production) tools and measurements are currently used in the preparation of (your) product?

Discussion on this question was kicked off by considering a list of measurement techniques identified in the 2013 TAPPI Cellulose nano-materials book: Production and Applications of Cellulose Nanomaterials, ${ }^{1}$ which can be mined for a great deal of information regarding ongoing research in CNM. Of interest here are data found in Tables 3 and 4 below. These tables are compilations of the imaging and characterization methods used by the researchers in individual articles within this resource.

Table 3: Most common measurement techniques for characterization of nanocellulose

\begin{tabular}{|l|}
\hline \multicolumn{1}{|c|}{$\begin{array}{c}\text { Top Methods for Imaging and Characterizing Cellulose } \\
\text { Nanomaterials }\end{array}$} \\
\hline Scanning Electron Microscopy (SEM) \\
\hline Transmission Electron Microscopy (TEM) \\
\hline Atomic Force Microscopy (AFM) \\
\hline Thermal Gravimetric Analysis (TGA) \\
\hline X-ray Diffraction (XRD) \\
\hline Raman Spectroscopy (RS) \\
\hline Fourier Transform Infrared (FTIR) Spectroscopy \\
\hline Ultraviolet-Visible Spectroscopy (UV/Vis) \\
\hline X-ray Photoelectron Spectroscopy (XPS) \\
\hline
\end{tabular}

Table 4: Alternative measurement techniques for characterization of nanocellulose

\begin{tabular}{|l|}
\hline \multicolumn{1}{|c|}{$\begin{array}{c}\text { Additional Methods for Imaging and Characterizing } \\
\text { Cellulose Nanomaterials }\end{array}$} \\
\hline Dynamic Light Scattering (DLS) \\
\hline Nuclear Magnetic Resonance (NMR) \\
\hline $\begin{array}{l}\text { Inductively Coupled Plasma Optical Emission } \\
\text { Spectroscopy (ICP-OES) }\end{array}$ \\
\hline Dynamic Mechanical Analysis (DMA) \\
\hline Optical Digital Image Correlation (DIC) \\
\hline Förster Resonance Energy Transfer (FRET) \\
\hline Laser Scanning Confocal Microscopy (LSCM) \\
\hline Helium Ion Microscope (HIM) \\
\hline
\end{tabular}


Understandably, many of these methods are closely related to those used for CNT research. Table 3 shows the top methods used in CNM research reported in the book with SEM, TEM, and AFM being the three most commonly used methods. Table 4 contains less common techniques that have also been applied to characterize CNM. ${ }^{1}$ More importantly, the techniques listed in Tables 3 and 4 represent research or laboratory grade instruments; few of these instruments/methods are high throughput, manufacturing grade techniques.

Some additional observations and thoughts regarding these techniques were raised by participants of the manufacturing breakout session. They pointed out that some additional techniques could be useful, such as Raman, FT-IR, UV-Vis (for quantifying lignin content), DLS, fiber size analysis, and confocal microscopy. A few techniques are considered too time consuming for routine monitoring of CNM production (TEM, SEM, AFM, TGA, X-ray PES, DMA, FRET, and Inverse Gas Chromatography). Optical methods have the speed needed for manufacturing quality control, but it is unclear if they have sufficient sensitivity. Finally, two additional measurements were identified as "of interest". The manufacturers present for the discussion stated that X-ray fiber size analysis and NMR-based surface area measurement warrant further investigation, but a good deal of research remains to be performed prior to implementation of these techniques.

Are automation and high throughput testing and characterization critical?

Automated testing and process monitoring are considered critical for large-scale manufacturing. An ideal system would include full automation, high sensitivity to process changes, and high precision.

Is accuracy required or is precision (repeatability) more important?

While performing accurate measurements is always beneficial, consensus seemed to be that precision is preferred over accuracy. Reliability in a measurement system is very important in a manufacturing environment along with sensitivity of the measurements to process changes.

What have we missed? Are there other crucial measurement concerns regarding CNM characterization?

Two items were identified as having been largely overlooked to date: surface characterization (chemistry) and methods for routine measurement of branching.

\section{Key recommendations identified by the Manufacturing Breakout Session:}

The manufacturing breakout session concluded that the greatest measurement need is highthroughput characterization of CNM geometry (both size and shape) and surface energy/chemistry. Most of the current techniques used to quantify these key properties are too expensive (in terms of both time and money) to be utilized for quality control. Additionally, any new, faster techniques that are developed should still maintain a high enough sensitivity/resolution to detect changes in CNM properties during production.

\subsection{Research and Development Breakout Session Summary}

\section{Co-Chairs: Johan Foster and Chelsea Davis}

A breakout session comprised primarily of research faculty and government researchers focused on research and development metrology needs for nanocellulose. Discussions touched on the various applications and fields of study in which CNM are being deployed and the characteristics that are expected to contribute to bulk properties of products incorporating CNM. Similar to the 
manufacturing session discussion reported in the previous section, the research and development breakout session was driven by several key questions:

- What do you need to do your R \& D job?

- What have you experienced with incoming CNM (as received) quality and what key properties must be measured?

- What tools and measurements are currently used to define and characterize the material/product being researched?

- Are there other crucial measurement concerns regarding CNM characterization?

What do you need to do your research and development job?

The needs for different research labs tend to fall into various categories depending on the products and applications being investigated. For nanocomposites-focused research, the three principle properties of interest are CNM surface chemistry, CNM/matrix interface, and bulk mechanical properties of the final composite system. Surface chemistry refers to the number and location of functional groups (primarily sulfates and hydroxyls) on the CNM surface. Interfacial characterization requires development of tools that allow measurement of interfacial strength as well as component surface energies. Bulk properties of final composites require correlation between nanoscale properties of the nanoreinforcement and macroscopic properties of the composite.

CNM have historically been employed as thickening agents and viscosity modifiers in commercial liquids ranging from paint to shampoo to ketchup. For rheologists interested in the further development of CNM for viscosity control, the critical characteristics that must be measured are nanoparticle dispersion, surface charge, and morphology (e.g., aspect ratio).

The same characteristics are important for researchers investigating CNM for enhanced barrier film applications such as light, oxygen, and water shielding. Additionally, quantification of any sizing or chemicals applied to the CNM is essential in the development of thin film applications.

Independent of a specific application or targeted research focus, two morphological questions were raised. For CNF, the degree or extent of branching is considered a significant factor that influences many key CNM properties. However, a reliable method to quantify the degree of branching in $\mathrm{CNF}$ has yet to be developed. Additionally, CNF tends to be a highly entangled material and researchers have expressed a desire to be able to quantify the extent of entanglement as a measure of quality. It was proposed that coarse-grained and atomistic modeling is a potential tool to address both degree of branching and entanglement measurements. Finally, the impact of amorphous or hemicellulose present in both CNF and CNC needs to be measured. Researchers in this breakout session suggested that modeling could be applied to address this problem as well. Certain types of imaging, some of which are listed in Table 3 might also be helpful in quantifying amorphous versus crystalline content.

What have you experienced with incoming CNM (as received) quality and what key properties must be measured?

The quality and key properties that must be measured prior to use of received CNM varied greatly depending on the source. For CNM produced "in house" in some academic laboratories, electron microscopy, charge titration, and dispersibility in various solvents are measured. For commercial bleached pulp, some researchers would like to be able to perform a chemical analysis. For the bleached pulp obtained from the Forest Products Lab, the sulfur content, ICP- 
OCS, and Raman spectroscopy (to measure crystallinity) are measured. Finally, for CNF commercial pulp, the hemicellulose content, viscosity, light transmittance, and morphology by electron microscopy are measured.

What tools and measurements are currently used to define and characterize the material/product being researched?

A host of techniques was listed by the participants in the research and development breakout session. These macroscopic techniques and applications include (in no particular order):

- Thermal expansion

- DSC/TGA

- DMTA

- Tensile/fracture

- Ballistics impact

- Rheology

- Tribology

- Optical properties

- Barrier properties

- Conductivity

- Biodegradability/Service Life

What critical CNM properties require new tools/measurements to define and characterize the product?

The CNM properties that require new measurement techniques of interest to the research and development community fall into two categories: morphology and surface properties. CNM morphology spans several length scales from atomic level details (e.g., chain packing and organization/structure of crystallinity) through the nano length scale (e.g., CNC size and shape) all the way to microstructures (e.g., the branching and entanglement of CNF). Additional morphological characterization of the level and structure of dispersion in liquid suspensions and solid nanocomposite systems are also needed. Certain methods were recommended as potential measurement tools to access these various morphological characteristics. Near-field Scanning Optical Microscopy (NSOM) and other advanced optics techniques such as super-resolution and Coherent Anti-Stokes Raman Spectroscopy (CARS) were discussed.

Surface properties, particularly surface energy and surface charge density, are other properties that require new measurement techniques. ${ }^{*}$

Finally, the development of more comprehensive modeling tools is required to allow for both predictive and confirmatory relationships that can be used in conjunction with experimental data to further our understanding of key CNM properties.

Are there other crucial measurement concerns regarding CNM characterization?

\footnotetext{
* It is important to consider the relevant terminology and measurement systems required to describe the surface properties of interest for a given CNM and desired application. Here, "surface energy" is used in a generic manner to encompass surface interactions that span several length scales from atomistic to sub-micrometer.
} 
A focus on predictive modeling (on the multi-scale) will allow identification of missed or (presently) unquantifiable parameters, standardization of data sets from various researchers and laboratories, and physical explanations of observed phenomena and mechanisms.

\section{Key recommendations identified by the Research and Development Breakout Session:}

To summarize the research and development breakout session, the three major applications of CNM being investigated are nanocomposite systems, viscosity modification, and barrier/thin film applications. To enable research-based advancement of these CNM applications, measurement systems must be developed or adapted to quantify surface properties and morphology of cellulosic nanomaterials. More specific, high resolution characterization techniques must be developed in order to quantify surface energy and spatially-resolved surface chemistry as well as morphological details such as degree of crystallinity and degree of branching (in CNF).

\subsection{Standards Breakout Session Summary}

Co-Chairs: World Nieh, András Vladár, and Aaron Forster

A charge was given to the NIST Cellulose Nanomaterials Workshop Standards Breakout Session participants to develop a list of the needs in metrologies and standards required for cellulose nanomaterials (i.e. what methods, materials and data are needed to do the job?). Standards were considered in three ways for this discussion. First, the idea of standards as a uniform test method or set of methodologies to enable reproducible measurements (over time and between laboratories) of key CNM properties was considered. Second, the community addressed the development of standards in the context of EH\&S for CNM in manufacturing and consumer products as well as end-of-life environmental concerns. Third, standards often include reference materials with known properties that can be used to verify and calibrate various measurement systems.

\section{Standard Testing Methods}

The standards community is primarily concerned with the application of standards as they relate to CNM production in industrial, large-scale manufacturing settings. It was stated that standards development efforts (both test methods and EH\&S regulations) should focus on wood-based cellulosic materials because wood-based fiber is currently the predominant, most likely raw material for large-scale cellulose nanomaterials (CNM). To enable the development of standard measurement techniques, the following CNM properties should be considered:

- Morphology:
- Particle size
- Particle size distribution
○ Geometry (e.g., aspect ratio for CNC)
$\circ$ Degree of branching ( $\mathrm{CNF}$ only)

- Color

- Aggregation behavior: sensitivity and dispersibility

- Surface charge and chemical functionality before and after surface modification

Additionally, there are a few additional properties specific to CNF or CNC. For CNF, overall surface charge and suspension viscosity need to be characterized. For CNC, degree of crystallinity and lignin content must also be considered. 
Standard test methods are important in providing quality assurance to customers. This should include routine testing protocols for incoming raw materials, test methods for production control and tests of key properties of the final products. Once standard test methods are established, manufacturers and customers can determine the properties that need to be tested and the standard methods to use to test these properties.

Test methods for manufacturing should be realistic for a manufacturing environment in which tests are performed by quality control technicians or lead operators. Instruments in these test methods should be simple, easy to operate, fast, reliable and affordable for a production facility. Development of combined measurement approaches where simple test methods (direct or indirect) deployed in manufacturing settings are validated or calibrated by more complex, timeconsuming measurement methods requiring expensive instruments should be of the highest priority. A few additional concerns raised by standards breakout session participants were that:

- Mechanical tests are not easy to implement in a manufacturing environment.

- There is a need for standards to determine shelf life. However, shelf life must first be defined (what characteristic(s) should be monitored and what degree of change of these characteristics will be used to determine the expiration date?).

- There is a need to develop methods to measure thermal stability and water susceptibility.

- Test method standards should include precision and accuracy of the test method.

\section{Environmental Health and Safety Standards}

A second area of need for standards is the characterization of CNM-enabled products that will be delivered to consumers. The following areas or properties must be addressed to enable a full set of CNM characterization and production standards:

- Quality of dispersion in different media (e.g., composites, liquid suspensions)

- Mapping surface charge and functionality before and after surface functionalization of nanomaterials

- Do CNM-containing products require special standard testing methods to address mechanical, thermal, and energy transport properties compared to traditional materials?

- Standards to support life cycle cost (including material degradation)

Environmental health and safety $(\mathrm{EH} \& \mathrm{~S})$ is another field of great concern to the standards community. Methods to measure CNM release or exposure for occupational safety and in the environment should be developed. Also, confirmatory detection methods for CNM will allow validation of EH\&S techniques. For example, how does one measure CNM concentrations in air and other media (especially on other cellulose surfaces, liquids, etc.)? Once detection methods have been developed and confirmed, do exposure limits need to be established? Are these exposure limits dependent on surface functionalization and the surrounding medium?

Tracking the life cycle of CNM in the environment is also an important role that the standards community must play prior to and during the industrialization of nanocellulose. Key features of the CNM life cycle that will need to be monitored include incorporation within consumer products, migration or release of nanomaterials from the surface, thermal or other environmental degradation, and the fate of CNM during recycling.

CNM toxicity experiments are required for legal manufacturing. Measurements should be made concerning: 
- Food applications

- Human inhalation, ingestion, dermal absorption, and neurotoxicity.

- Potential mutagenicity and carcinogenicity

Micro-scale and bulk cellulose are widely incorporated in many consumer and industrial products. The EH\&S regulations for larger cellulosic material are well established and it is generally accepted to be a benign material in every sense of the word. However, questions have been raised regarding the nanoscopic size of CNM. The smaller particles could potentially be absorbed more easily into the body and be transported throughout the body. Also, the surface functionalization of nanocellulose is potentially of a greater concern than in microcellulose due to the exponentially greater fraction of surface area per unit mass inherent to all nanomaterials. Future studies that bridge length scales between existing studies on conventional (macro- and micro-scale) cellulose to new experiments on CNM are required.

\section{CNM Reference Materials}

The participants of the standards breakout session discussed not only reference materials that need to be developed but also the properties and varieties of these potential materials. Prior to the development of any standard materials, the properties and techniques discussed in this report must be established. Reference materials for the purpose of size measurements, for both unaggregated and aggregated CNM are needed. It was suggested that CNM reference materials be derived from TEMPO CNF rather than the $\mathrm{H}_{2} \mathrm{SO}_{4}$ process. Additionally, any CNM reference materials must be stable (long shelf life) and applicable to the measurement of multiple properties.

In addition to reference materials, reference data should also be collected, curated, and published in order to accomplish many of the same functions as RM. A measurement matrix of CNM properties measured for different manufacturing methods, source materials, and suppliers should be established.

\section{Key recommendations identified by the Standards Breakout Session:}

In summary, the standards community is working on a three-pronged approach to CNM industrialization. To enable consistency and quality control in manufacturing, standard testing methods for key properties of CNM must be established. EH\&S studies focused on CNM toxicity (for both manufacturing and commercial product release) as well as environmental impact must be conducted prior to large-scale production. Finally, reference materials, such as those offered by NRC-Canada must be utilized to facilitate collaboration between academia and industry. 


\section{Conclusion}

Cellulose nanomaterials are of great interest to many materials researchers and engineers in fundamental research (academia and government institutes), industry, and the standards community. The potential of CNM as a sustainable and potentially non-hazardous nanomaterial additive in composite systems, industrial processing fluids, and barrier films is currently hindered by the lack of reliable, industrially relevant measurement tools required to control and verify uniformity of (1) geometry, (2) chemistry, (3) processing, and (4) environmental health impacts. Naturally derived materials have a much greater inherent variation, due to the various sources from which they are obtained and the manner in which they are processed.

CNM morphology and surface characteristics are the two general sets of properties that need to be quantified to address quality control. Morphology encompasses geometric properties such as length, width, shape, and aspect ratio. It also refers to degree of crystallinity and polymer chain configuration within cellulose nanocrystals. For CNF, morphological concerns such as extent of branching and degree of entanglement also persist. Surface properties that need to be measured include surface sulfur content (for wood-derived, $\mathrm{H}_{2} \mathrm{SO}_{4}$ processed $\mathrm{CNM}$ ), surface energy and dispersibility in various media.

While these general properties must be measured for development of commercial applications, several other measurement requirements have to be addressed before full-scale industrial production of CNM can occur. Quality measurements need to be in line, high throughput, and easy to use to facilitate manufacturing process monitoring. Many of the current techniques used to quantify these key properties are too expensive. Additionally, new, faster techniques should still maintain sufficient sensitivity and resolution to detect changes in CNM properties during production. An additional concern raised by several speakers during the MNCNM Workshop concerned the drying, shipping, and redispersion of CNM. Shipping process slurries is expensive compared to powders, but the current freeze and spray drying techniques lead to severe aggregation. These aggregation processes inhibit facile resuspension of CNM in liquid media prior to incorporation into other products.

The standards community representatives highlighted the need to address several unanswered questions regarding the safe and reproducible use of CNM during their breakout discussion. Measurement systems that allow the detection of ultra-low concentrations of cellulose nanoparticles are not widely available. The ability to measure CNM concentrations in air, water, and soil will enable standardization of toxicity limits through controlled studies. Additionally, environmental impact monitoring can only be achieved through effective detection of CNM. Concerns were also raised by participants in the standards breakout session regarding the shelf life of any CNM reference materials developed and distributed to calibrate surface energy or morphology measurements. Since $\mathrm{CNM}$ is an inherently biodegradable material, degradation and expiration dates must be considered during development of these standards.

The three major applications of CNM that are a priority for research and development are nanocomposite systems, viscosity modifiers, and barrier/thin film applications. Measurement systems must be developed or adapted to quantify surface properties and morphology of cellulosic nanomaterials. Cellulosic nanomaterials are bio-derived engineering materials that have the potential to rejuvenate the pulp and paper industry and change the economics of commodity industries. As nanoreinforced composite materials become ubiquitous in structural and consumer products, sustainable material sources will play a larger role in materials development. The key physical properties and metrology needs identified by the participants of this workshop and highlighted in this report are important factors in the commercialization of CNM. 
9 References

(1) Postek, M. T.; Rudie, A. W.; Moon, R. J.; Bilodeau, M. A. Production and Applications of Cellulose Nanomaterials; 1st ed.; TAPPI Press: Peachtree Corners, GA, 2013.

(2) Samir, M. A. S. A.; Alloin, F.; Dufresne, A. Review of Recent Research into Cellulosic Whiskers, Their Properties and Their Application in Nanocomposite Field.

Biomacromolecules 2005, 6, 612-626.

(3) Cowie, J.; Bilek, E. M.; Wegner, T. H.; Shatkin, J. A. Market Projections of Cellulose Nanomaterial-Enabled Products - Part 2: Volume Estimates. Tappi J. 2014, 13, 57-69.

(4) Shatkin, J. A.; Wegner, T. H.; Bilek, E. M.; Cowie, J. Market Projections of Cellulose Nanomaterial-Enabled Products - Part 1: Applications. TAPPI J. 2014, 13, 9-16.

(5) Moon, R. J.; Martini, A.; Nairn, J.; Simonsen, J.; Youngblood, J. P. Cellulose Nanomaterials Review: Structure, Properties and Nanocomposites. Chem. Soc. Rev. 2011, 40, 3941-3994.

(6) Chin, J.; Byrd, E.; Embree, N.; Garver, J.; Dickens, B.; Finn, T.; Martin, J. Accelerated UV Weathering Device Based on Integrating Sphere Technology. Rev. Sci. Instrum. 2004, 75, 4951-4959.

(7) Biyani, M. V.; Jorfi, M.; Weder, C.; Foster, E. J. Light-Stimulated Mechanically Switchable, Photopatternable Cellulose Nanocomposites. Polym. Chem. 2014, 5, 57165724.

(8) Biyani, M. V.; Weder, C.; Foster, E. J. Photoswitchable Nanocomposites Made from Coumarin-Functionalized Cellulose Nanocrystals. Polym. Chem. 2014, 5, 5501-5508.

(9) Biyani, M.; Foster, E. J.; Weder, C. Light-Healable Supramolecular Nanocomposites Based on Modified Cellulose Nanocrystals. ACS Macro Lett. 2013, 2, 236-240.

(10) VanderHart, D.; Atalla, R. Studies of Microstructure in Native Celluloses Using SolidState Carbon-13 NMR. Macromolecules 1984, 17, 1465-1472.

(11) Wickholm, K.; Larsson, P. T.; Iversen, T. Assignment of Non-Crystalline Forms in Cellulose I by CP/MAS 13 C NMR Spectroscopy. Carbohydr. Res. 1998, 312, 123-129.

(12) Hamad, W. Y.; Hu, T. Q. Structure-Process-Yield Interrelations in Nanocrystalline Cellulose Extraction. Can. J. Chem. Eng. 2010, 88, 392-402.

(13) Pan, J.; Hamad, W.; Straus, S. K. Parameters Affecting the Chiral Nematic Phase of Nanocrystalline Cellulose Films. Macromolecules 2010, 43, 3851-3858. 
(14) Lemke, C. H.; Dong, R. Y.; Michal, C. a.; Hamad, W. Y. New Insights into NanoCrystalline Cellulose Structure and Morphology Based on Solid-State NMR. Cellulose 2012, 19, 1619-1629.

(15) Sacui, I. A.; Nieuwendaal, R. C.; Burnett, D. J.; Stranick, S. J.; Jorfi, M.; Weder, C.; Foster, E. J.; Olsson, R. T.; Gilman, J. W. J. W. Comparison of the Properties of Cellulose Nanocrystals and Cellulose Nanofibrils Isolated from Bacteria, Tunicate, and Wood Processed Using Acid, Enzymatic, Mechanical, and Oxidative Methods. ACS Appl. Mater. Interfaces 2014, 6, 6127-6138.

(16) Canada, N. R. C. Certified Reference Materials Produced by NRC http://www.nrccnrc.gc.ca/eng/solutions/advisory/crm/inorganic/list_products.html.

(17) Brunauer, S.; Emmett, P. H.; Teller, E. Adsorption of Gases in Multimolecular Layers. $J$. Am. Chem. Soc. 1938, 60, 309-319.

(18) Diaz, J. a; Wu, X.; Martini, A.; Youngblood, J. P.; Moon, R. J. Thermal Expansion of Self-Organized and Shear-Oriented Cellulose Nanocrystal Films. Biomacromolecules 2013, 14, 2900-2908.

(19) Padalkar, S.; Capadona, J. R.; Rowan, S. J.; Weder, C.; Moon, R. J.; Stanciu, L. a. SelfAssembly and Alignment of Semiconductor Nanoparticles on Cellulose Nanocrystals. $J$. Mater. Sci. 2011, 46, 5672-5679.

(20) Saito, T.; Nishiyama, Y.; Putaux, J.-L.; Vignon, M.; Isogai, A. Homogeneous Suspensions of Individualized Microfibrils from TEMPO-Catalyzed Oxidation of Native Cellulose. Biomacromolecules 2006, 7, 1687-1691.

(21) Isogai, A.; Kato, Y. Preparation of Polyuronic Acid from Cellulose by TEMPO-Mediated Oxidation. Cellulose 1998, 5, 153-164. 
10 Appendix

\subsection{Workshop Agenda}

NIST Workshop on Measurement Needs for Cellulose Nanomaterials Fairmont Hotel, Vancouver, British Columbia

Monday June 23, 2014

8:00 AM Registration and Coffee

8:20 AM Welcome and Introductory Remarks - Jeff Gilman

Morning Presentations (10 min each) Followed by Discussion

8:30 AM Alan Rudie (Supervisory Research Chemist, Forest Products Laboratory -USDA)

Kim Nelson (Vice President, American Process, Inc.)

Robert Moon (Materials Research Engineer, Forest Products Laboratory -USDA)

Sean Ireland (Manager, New Technologies \& Market Ventures, Verso Paper)

Linda Johnston (Team Lead, Nanoscale Measurement, Measurement Science and

9:20 AM Q \& A

Standards, National Research Council Canada)

10:00 AM Coffee Break

10:15 AM Wadood Hamad (Principal Scientist, FPInnovations)

Mike Bilodeau (Associate Director, University of Maine Forest Bioproducts Research Initiative)

Johan Foster (Senior Scientist, Adolphe Merkle Institute)

Jo Anne Shatkin (President, Vireo Advisors, LLC)

Aaron Forster (Materials Research Engineer, NIST)

11:05 AM Q \& A

11:45 AM Working Lunch (provided) in Breakout Session Rooms

Breakout Sessions (Concurrent Sessions)

12:00 PM Parameters of Interest and Measurement Goals

Manufacturing Session

Facilitated by Mike Postek (NIST) and Alan Rudie (FPL/USDA)

R\&D Session

Facilitated by Chelsea Davis (NIST) and Johan Foster (Fribourg)

Standards Session

Facilitated by Aaron Forster (NIST), András Vladár (NIST), and World Nieh (USDA)

2:00 PM Coffee Break

Reports \& Closing Remarks (reassemble)

2:30 PM Breakout Session Reports - Jeff Gilman

2:45 PM Manufacturing Breakout Summary - Alan Rudie

3:15 PM Research and Development Breakout Summary - Johan Foster

3:45 PM Standards Breakout Summary - World Nieh

4:30 PM Concluding Remarks \& Wrap Up

5:00 PM Adjourn 


\subsection{List of Workshop Participants}

Umesh Agarwal - US Department of Agriculture Forestry Service

Lars Axrup - Stora Enso

Michael Bilodeau - University of Maine

Jean Bouchard - FPInnovations

Chelsea Davis - National Institute of Standards and Technology

Ulla Forsstrom - VTT

Aaron Forster - National Institute of Standards and Technology

Johan Foster - KTH

Jeffrey Gilman - National Institute of Standards and Technology

Dave Haldane - Mainline

Sean Ireland - Verso Paper

Donna Johnson - University of Maine

Linda Johnston - National Research Council (Canada)

Henri Kastinen - Storaenso

Alfred Leung - National Research Council (Canada)

Robert Moon - US Department of Agriculture Forestry Service

Hiroaki Namba - Nippon Paper Group

Kimberly Nelson - American Process, Inc.

Phil Nelson - Uniquest

World Nieh - US Department of Agriculture

Sunkyu Park - North Carolina State University

Michael Postek - National Institute of Standards and Technology

Alan Rudie - US Department of Agriculture Forestry Service

Shinji Sato - Nippon Paper Group

Jo Anne Shatkin - Vireo Advisors, LLC

David Skuse - Imerys

Brandon Van Leer - FEI

András Vladár - National Institute of Standards and Technology

Yi-Ting Wang - University of Illinois at Urbana-Champaign

Theodore Wegner - US Department of Agriculture Forestry Service Junyong Zhu - US Department of Agriculture Forestry Service 


\subsection{ISO TC 229 Project 19716 - Characterization of Cellulose Nanocrystals}

\section{Scope}

This Technical Report reviews commonly used methods for the characterization of cellulose nanocrystals (CNCs), including sample preparation, measurement methods and data analysis. Selected measurands that are important for characterization of CNCs for commercial production and applications are covered. These include CNC composition (crystallinity, surface functional groups, degree of polymerization and contaminants), morphology as assessed by microscopy and light scattering methods, zeta potential, specific surface area, viscosity and thermal stability.

\section{ISO TC 229 Project 19716 Contents*}

1. Scope

2. Terms and definitions

3. Symbols (and abbreviated terms)

4. Production of Cellulose Nanocrystals

5. Composition

5.1. Chemical identity

5.2. Crystallinity

5.2.1. X-ray diffraction

5.2.2. NMR

5.2.3. Vibrational spectroscopy

5.2.4. Crystallinity measurements for $\mathrm{CNCs}$

5.3. Degree of polymerization

5.4. Surface chemistry

5.4.1. Determination of sulfate half-esters

5.4.2. Determination of carboxylic acids

5.5. Moisture content

5.6. Contaminants

5.6.1. Determination of residual impurities derived from cellulosic biomass

5.6.2. Determination of metal ions by inductively coupled plasma-mass spectrometry

5.6.3. XPS for detection of contaminants

6. CNC Morphology

6.1. Distribution of length and cross-section from microscopy

6.1.1. Electron microscopy

6.1.2. Atomic force microscopy

6.1.3. Image analysis considerations

6.1.4. Microscopy size distributions for CNCs

6.2. Size measurement by dynamic light scattering

7. CNC Surface Characteristics

7.1. Specific surface area

8. Zeta potential

9. Other Measurands

9.1. Thermal stability

9.2. Viscosity

10. Concluding comments

Bibliography

*Draft in progress (not final version). 


\subsection{Nano EH\&S Roadmap}

This version of the EH\&S roadmap for cellulose nanomaterials is based on three prior assessments:

- Evaluation by the National Institute for Occupational Safety and Health (NIOSH)

- A life cycle risk analysis (LCRA) by Vireo Advisors, LLC which evaluated the available data and identified/prioritized gaps for occupational, environmental, and consumer safety

- Assessment by $\mathrm{P}^{3} \mathrm{Nano}$, in collaboration with Vireo Advisors of data availability and gaps to complete a Safety Data Sheet (SDS) compliant with the Globally Harmonized Standard (GHS) format, soon to be required by the Occupational Health and Safety Administration (OSHA).

Identified below are Data Gaps and priorities for both CNC and CNF, collectively referred to as cellulose nanomaterials.

\section{Safe Exposure Levels}

Currently there are no established and vetted occupational exposure limits specific to cellulose nanomaterials and engineered cellulose nanomaterials. Preliminary data indicate that cellulose nanomaterials are bio-durable in artificial lung fluid even after one year of exposure. In addition, preliminary in vitro studies have indicated that these materials can cause both inflammation and hypertrophy. Based on current information, the primary potential hazard for an occupational user currently associated with cellulose nanomaterials is the inhalation of pure, dried material during laboratory research and development and commercial production and manufacturing.

In order to determine the potential for health effects with inhalation exposure to cellulose nanomaterials, both NIOSH and many other partners are currently performing in vivo inhalation research on both fibrils and crystals. As with similar materials of this type, it is good practice to control potential releases by making use of engineering controls (such as local exhaust ventilation or hoods and PPE) during the handling of dry product or manipulation of product composites until clear guidance on acceptable exposure concentrations is established.

A summary of preliminary recommendations received from NIOSH by the USDA Forest Service Forest Products Laboratory to maintain a safe and healthy workplace includes:

1. Respiratory protection should be utilized during the removal of dried product from trays and during the cutting and milling of CNC composites. Note: respirator use was not recommended as the primary form of employee respiratory protection.

2. Aerosolization of product during the handling CNMs. Gloves, laboratory coats with elastic wristbands, and face shields/safety glasses are recommended to decrease potential for dermal or eye exposure. Inhalation exposure can be decreased by the use of local exhaust ventilation (LEV).

3. Consider increasing the airflow into the portable fume extractor unit or moving the hood closer to the workbench during drying operations to improve capture efficiency. For controlling exposures using a LEV system, the placement of the hood near the source is critical. Capture velocity drops by approximately $90 \%$ within one duct diameter $(15 \mathrm{~cm}$ to $20 \mathrm{~cm})$ from the hood face. 
4. Work areas should be cleaned following a spill, task, and/or at the end of each work shift (at a minimum) using a HEPA-filtered vacuum either cleaner or wet wiping methods. Dry sweeping or air hoses should not be used to clean work areas as the re-suspension of particles can occur.

5. Transfer of product outside of the production area should be minimized.

The potential hazards identified by NIOSH at each life cycle stage are listed in Table 2.

To move the safe handling and use of cellulosic nanomaterials forward most expeditiously, the following needs to be accomplished:

- Development of a Cellulose Nanomaterials Safety Data Sheet populated with available information to identify gaps;

- Development of a network of credible collaborating organizations to advance environmental health and safety research on cellulose nanomaterials;

- Coordination of efforts nationally and internationally to develop credible and reliable detection methods for cellulose nanomaterials;

- Coordination of external parties nationally and internationally to assist in establishing credible safe workplace environments;

- Development of effective and efficient methods and protocols for monitoring cellulose nanomaterials in the workplace;

- Development of a scope of work to compare the safety of cellulose nanomaterials to currently used cellulosic materials for food-related applications;

- Coordinate external efforts regarding the environmental impacts of cellulose nanomaterials;

- Selectively conduct full life-cycle assessments to quantify cellulose nanomaterials environmental performance for specific product applications. Below, data gaps and priorities for both $\mathrm{CNC}$ and $\mathrm{CNF}$, collectively referred to as cellulose nanomaterials, are identified from a risk assessment performed by Vireo Advisors in 2013. These data gaps consider occupational, consumer and environmental exposure over the product life cycle, as well as analyze available data versus data requirements for developing Safety Data Sheets for CNC and CNF compliant with the Globally Harmonized Standard.

- In 2013, Vireo Advisors conducted a Life Cycle Risk Assessment (LCRA) to identify potential risks and data gaps for five products using CNM on behalf of the US Forest Service Forest Products Laboratory. The findings of the LCRA form the foundation of a Roadmap to inform the CNM EH\&S and its environmental disposition that will guide the safe development of products and address stakeholder requirements for demonstration of safe handling. Demonstration of environmental benefits and sustainability performance would advance the commercial potential of CNM for environmentally preferred product designations, bio-based chemicals, as well as end-user, customer and consumer requirements.

\section{Measurement}

Measurement needs are prioritized in the EH\&S roadmap because, without them, none of the other needed studies can be conducted in a reliable way. This includes confirmatory detection techniques, quantitative measurements, and standardized protocols for sampling and analysis in environmental media, including air, water, and solid matrices.

Measurement need-1) confirmatory material detection in environmental media

Currently SEM - time and labor intensive, costly; does not work well in most matrices M1.1 aerosolized dust 
Necessary for occupational exposure measurements

M1.2 aqueous solutions

Required for effluent testing, as well as confirmatory presence of material in feedstock.

M1.3 non-aqueous solutions

Other liquid forms that may be used to deliver CNM.

M1.4 solid matrices (e.g., composites, biological matrices)

Measurement need-2) quantitative measurement techniques for environmental media that preferably do not rely on electron microscopy, particularly for aerosol measurements that would take place in a manufacturing environment.

M2.1 aerosolized dust

M2.2 aqueous solutions

M2.3 non-aqueous solutions

M2.4 solid matrices (e.g., composites, biological matrices)

Measurement need-3) standard sampling methods to assess occupational exposure

Measurement need-4) standard methods for other media

\section{Physical-Chemical Data}

The assessment of safety for nanomaterials requires better characterization of key properties that may influence biological and environmental behavior. These measurements are not required only for dry and aqueous solutions, but also for as-delivered forms in toxicology experiments to measure dose. Measures include:

PC1) Standardized measurement and reporting techniques for CNM.

There are as yet no standardized measurements of nanoscale materials. The data needed here are the appropriate measurement techniques for cellulose nanomaterials as raw materials, describing the size, size distribution, shape, aspect ratio, level of branching, aggregation state, porosity, surface chemistry, surface charge, crystallinity, and dustiness.

PC2) Flammability

Cellulose is known be combustible at elevated temperatures $\left(240{ }^{\circ} \mathrm{C}\right)$ but the difference in flammability for cellulose nanomaterials is unknown.

PC3) Explosivity

Cellulose dust is known to be explosive, with a deflagration index (Kst) value of 229, which puts it in a Strong Explosive category. Nanomaterials may pose a greater explosion hazard due a higher ratio of surface area to mass, and therefore need to be assessed. Work is currently under way at NIOSH to investigate the flammability and explosivity of CNF and CNC produced by the Forest Products Laboratory. There is a need to test other materials so a range of values can be developed.

PC4) Incompatibility with other substances

Cellulose dust is incompatible with oxidizing substances. It remains unknown whether there are additional incompatible materials due to the greater reactivity of nanoscale surfaces.

\section{Human Health}

Demonstration of safety of cellulose nanomaterials.

A variety of existing short-term (acute) toxicity test methods for testing chemicals was used to assess $\mathrm{CNC}$ and $\mathrm{CNF}$, as were some novel assays. Most of these were in vitro assays. However, it is not clear whether any of the assays are appropriate for testing nanomaterials generally, or CNM specifically. There is a need to confirm the methods work for the stated purpose, as some standard toxicity tests have been shown to give false results due to interference of the 
nanoparticles with test reagents or media. The OECD has been evaluating its standard methods for applicability to nanomaterials. These methods need to be reviewed and considered in terms of their ability to report on the safety of CNM.

\section{Bridging studies.}

Bridging studies that link existing whole animal (in vivo) studies to in vitro tests are an opportunity to further develop in vitro methods for future assessments. First, there is a need for in vivo testing of $\mathrm{CNC}$ and $\mathrm{CNF}$ to evaluate whether they behave similarly to other forms of cellulose that are currently used in food and medical applications.

Current evidence is inadequate for this demonstration. Oral exposure studies are considered high priority because 1) several forms of cellulose are already approved by FDA and others for use as food additives and food contact substances, or are grandfathered as Generally Regarded as Safe (GRAS); 2) assessing whether novel nano-forms behave differently from approved forms has potential to open food-contact applications-best to compare a range of studies and have peer review of the studies; 3) this work is prioritized because further animal testing will not be required if differences in uptake are not observed. 4) a sound comparison of in vitro tests with in vivo (whole animal) bioassays will create an easier path forward for surface-functionalized and materials produced by alternate pathways to be assessed for safety. It will allow for bridging studies, so that animal testing need not be repeated for each surface modification.

\section{Inhalation}

Inhalation exposure studies are needed because all foreign particles are potentially hazardous to the lung, and there are no nano-specific requirements for workplace safety. There is a need to assess the effects of inhalation exposure in the workplace. Further, additional endpoints should be studied to demonstrate overall safety in a breadth of uses. Long-term exposure studies are needed.

HH1) Assess oral exposure to CNM in comparison to already approved and Generally Regarded as Safe cellulosic substances in food and drugs.

HH1.1 Subchronic oral feeding study (28 d) with variety of CNM (CNC, CNF) and approved/GRAS food additives

HH1.2 Assess adsorption, distribution, metabolism and excretion (ADME) of CNM

HH1.3 Classical endpoint measurements (e.g., mutagenicity, immunotoxicity)

HH1.4 Assess use of in vitro techniques for alternate surface functionalized forms - to allow these studies to be used in the future for determination of safety of novel forms

$\mathrm{HH} 2$ ) Dermal exposure studies

Ensure safety by skin contact (skin sensitization, irritation studies).

HH3) Assess safety via inhalation

See NIOSH, above. Studies need to include valid protocols for nanoscale materials. For example, a challenge is to aerosolize CNM in high enough concentrations to adhere to standard testing protocols.

HH4) Systemic toxicity testing

To date, no studies exist that have explored the following endpoints in animal systems: neurotoxicity, carcinogenicity and reproductive/developmental toxicity

\section{Environmental Safety}

While CNM is presumed to be a low hazard to the environment, studies are needed to demonstrate this. The following categories of information are needed. 
ENV1) biodegradation testing

Few data exist to report the biodegradation rates of CNM. Available data suggest that some forms are degraded more rapidly in standardized tests.

ENV2) Aquatic and marine organism testing

Some forms of CNM have been tested in a diversity of standardized and non-standard aquatic toxicity tests. More thorough studies are needed to complete the demonstration of safety.

ENV3) Life Cycle Assessment

Environmental claims must be substantiated with data to demonstrate the safety and sustainability not only of the CNM, but also of its production process and use in products. Doing so would facilitate entry into more markets based on environmental performance 